\title{
Drell-Yan production at the LHC in a four site Higgsless model
}

\author{
Elena Accomando ${ }^{(a)}$, Stefania De $\operatorname{Curtis}^{(a)}$, Daniele Dominici ${ }^{(a, b)}$ and Luca Fedeli ${ }^{(b)}$ \\ (a) INFN, 50019 Sesto F., Firenze, Italy, (b) Department of Physics, \\ University of Florence, 50019 Sesto F., Firenze, Italy.
}

\begin{abstract}
We consider a four site Higgsless model based on the $S U(2)_{L} \times S U(2)_{1} \times S U(2)_{2} \times$ $U(1)_{Y}$ gauge symmetry, which predicts two neutral and four charged extra gauge bosons, $Z_{1,2}$ and $W_{1,2}^{ \pm}$. We compute the properties of the new particles, and derive indirect and direct limits on their masses and couplings from LEP and Tevatron data. In contrast to other Higgsless models, characterized by fermiophobic extra gauge bosons, here sizeable fermion-boson couplings are allowed by the electroweak precision data. The prospects of detecting the new predicted particles in the favoured Drell-Yan channel at the LHC are thus investigated. The outcome is that all six extra gauge bosons could be discovered in the early stage of the LHC low-luminosity run.
\end{abstract}

\section{INTRODUCTION}

During the last years a remarkable activity has been devoted to investigate Higgsless models [1, 2, 3, 4, 5, 6, 7, 8, 9] because they emerge in a natural way when considering local gauge theories in five dimensions (5D). Their major outcome consists in delaying the unitarity violation of vector boson scattering (VBS) amplitudes to higher energies compared to the answer of the Standard Model (SM) without a light Higgs, via the exchange of KaluzaKlein (KK) excitations [10]. The discretization of the compact fifth dimension to a lattice generates the so-called deconstructed theories which are chiral lagrangians with a number of replicas of the gauge group equal to the number of lattice sites [11, 12, 13, 14, 15, 16, 17, 18, 19]. Models have been proposed, assuming a $S U(2)_{L} \times S U(2)_{R} \times U(1)_{B-L}$ gauge group in the $5 \mathrm{D}$ bulk, $[1,[2, \underline{3}, 4,4, \underline{6}, \mathbf{7}, \mathbf{8}, \underline{9}]$, in the framework suggested by the AdS/CFT correspondence, or also with a simpler gauge group $S U(2)$ in the bulk [20, 21, 22, 23, 24].

The drawback of all these models, as with technicolor theories, is to reconcile the presence of a relatively low KK-spectrum, necessary to delay the unitarity violation to TeV-energies, with the electroweak precision tests (EWPT) whose measurements can be expressed in terms of the $\epsilon_{1}, \epsilon_{2}$ and $\epsilon_{3}$ (or $\left.T, U, S\right)$ parameters. More in detail, while $\epsilon_{1}$ and $\epsilon_{2}$ are protected by 
the custodial symmetry [25, 26, 27, 28], shared by both the aforementioned classes of models, the $\epsilon_{3}(S)$ parameter constitutes the real obstacle to EWPT consistency. This problem can be solved by either delocalizing fermions along the fifth dimension [7, 29] or, equivalently in the deconstructed version of the model, by allowing for direct couplings between new vector bosons and SM fermions [30]. In the simplest version of this latter class of models, corresponding to just three lattice sites and gauge symmetry $S U(2)_{L} \times S U(2) \times U(1)_{Y}$ (the so-called BESS model [31, 32]), the requirement of vanishing of the $\epsilon_{3}$ parameter implies that the new triplet of vector bosons is almost fermiophobic. As a consequence, the only production channels where the new gauge bosons can be searched for are those driven by boson-boson couplings. The Higgsless literature has been thus mostly focused on difficult multi-particle processes which require high luminosity to be detected, that is vector boson fusion (VBF) and associated production of new gauge bosons with SM ones [33, 34, 35].

We extend the minimal three site model by inserting an additional lattice site. This new four site Higgsless model, based on the $S U(2)_{L} \times S U(2)_{1} \times S U(2)_{2} \times U(1)_{Y}$ gauge symmetry, predicts two neutral and four charged extra gauge bosons, $Z_{1,2}$ and $W_{1,2}^{ \pm}$, and satisfies the EWPT constraints without necessarily having fermiophobic resonances. Within this framework, the more promising Drell-Yan processes become particularly relevant for the extra gauge boson search at the LHC.

In Section II we review the main properties of the model, in particular we derive the couplings of new gauge bosons to SM fermions. In Section [II, we discuss the bounds on masses and couplings coming from EWPT and partial wave unitarity requirement. In Section IV, we discuss the prospects of detection of the new particles in Drell-Yan channels at the LHC, then we give out our conclusions. In Appendix A, we compute the spectrum of the new gauge bosons and in Appendix B we list numerical values corresponding to the considered scenarios.

\section{REVIEW OF THE MODEL}

The class of models we are interested in follows the idea of dimensional deconstruction [11, 12, 13, 14] and was recently studied in [30]. The so-classified theories can also be seen as generalizations of the BESS model [31, 32, 36] to an arbitrary number of new triplets of gauge bosons. In their general formulation [20, 21, 22, 23, 24], they are based on the 
$S U(2)_{L} \otimes S U(2)^{K} \otimes U(1)_{Y}$ gauge symmetry, and contain $K+1$ non linear $\sigma$-model scalar fields $\Sigma_{i}, i=1, \cdots, K+1$, transforming as

$$
\begin{aligned}
& \Sigma_{1} \rightarrow L \Sigma_{1} U_{1}^{\dagger}, \\
& \Sigma_{i} \rightarrow U_{i-1} \Sigma_{i} U_{i}^{\dagger}, \quad i=2, \cdots, K, \\
& \Sigma_{K+1} \rightarrow U_{K} \Sigma_{K+1} R^{\dagger},
\end{aligned}
$$

with $U_{i} \in S U(2), L \in S U(2)_{L}, R \in U(1)_{Y}$. The related Lagrangian for scalars and gauge fields is given by

$$
\mathcal{L}=\sum_{i=1}^{K+1} f_{i}^{2} \operatorname{Tr}\left[D_{\mu} \Sigma_{i}^{\dagger} D^{\mu} \Sigma_{i}\right]-\frac{1}{2} \sum_{i=1}^{K} \operatorname{Tr}\left[\left(F_{\mu \nu}^{i}\right)^{2}\right]-\frac{1}{2} \operatorname{Tr}\left[\left(F_{\mu \nu}(\tilde{W})\right)^{2}\right]-\frac{1}{2} \operatorname{Tr}\left[\left(F_{\mu \nu}(\tilde{Y})\right)^{2}\right],
$$

with the covariant derivatives defined as follows

$$
\begin{gathered}
D_{\mu} \Sigma_{1}=\partial_{\mu} \Sigma_{1}-i \tilde{g} \tilde{W}_{\mu} \Sigma_{1}+i \Sigma_{1} g_{1} \tilde{A}_{\mu}^{1}, \\
D_{\mu} \Sigma_{i}=\partial_{\mu} \Sigma_{i}-i g_{i-1} \tilde{A}_{\mu}^{i-1} \Sigma_{i}+i \Sigma_{i} g_{i} \tilde{A}_{\mu}^{i}, \quad i=2, \cdots, K \\
D_{\mu} \Sigma_{K+1}=\partial_{\mu} \Sigma_{K+1}-i g_{K} \tilde{A}_{\mu}^{K} \Sigma_{K+1}+i \tilde{g}^{\prime} \Sigma_{K+1} \tilde{Y}_{\mu},
\end{gathered}
$$

where $\tilde{A}_{\mu}^{i}=\tilde{A}_{\mu}^{i a} \tau^{a} / 2$ and $g_{i}$ are the gauge fields and gauge coupling constants associated to the groups $G_{i}, i=1, \cdots, K ; \tilde{W}_{\mu}=\tilde{W}_{\mu}^{a} \tau^{a} / 2, \tilde{Y}_{\mu}=\tilde{\mathcal{Y}}_{\mu} \tau^{3} / 2$ and $\tilde{g}, \tilde{g}^{\prime}$ are the gauge fields and couplings associated to $S U(2)_{L}$ and $U(1)_{Y}$ respectively, and $f_{i}$ are $K+1$ free parameters, the link coupling constants. In the continuum limit, different $f_{i}$ can describe a generic warped metric, while the flat case can have a correspondence with a scenario where all $f_{i}$ are equal.

Direct couplings of new gauge bosons to SM fermions can be included in a way that preserve the symmetry of the model. The fermion Lagrangian is given by [30]

$$
\begin{aligned}
\mathcal{L}_{\text {fermions }} & =\bar{\psi}_{L} i \gamma^{\mu} \partial_{\mu} \psi_{L}+\bar{\psi}_{R} i \gamma^{\mu} \partial_{\mu} \psi_{R} \\
& -\frac{1}{1+\sum_{i=1}^{K} b_{i}} \bar{\psi}_{L} \gamma^{\mu} \tilde{g} \tilde{W}_{\mu} \psi_{L} \\
& -\sum_{i=1}^{K} \frac{b_{i}}{1+\sum_{j=1}^{K} b_{j}} \bar{\psi}_{L} \gamma^{\mu} g_{i} \tilde{A}_{\mu}^{i} \psi_{L} \\
& -\bar{\psi}_{R} \gamma^{\mu}\left(\tilde{g}^{\prime} \tilde{Y}_{\mu}+\frac{1}{2} \tilde{g}^{\prime}(B-L) \tilde{\mathcal{Y}}_{\mu}\right) \psi_{R}-\bar{\psi}_{L} \gamma^{\mu} \frac{1}{2} \tilde{g}^{\prime}(B-L) \tilde{\mathcal{Y}}_{\mu} \psi_{L}
\end{aligned}
$$

where $b_{i}$ are arbitrary dimensionless parameters, which we assume universal, and $\psi_{L(R)}$ denote the standard quarks and leptons. Direct couplings of the new gauge bosons to the 
right-handed fermions can also be introduced but they are strongly constrained by data from non-leptonic K-decays and $b \rightarrow s \gamma$ processes [37] to be of order $10^{-3}$ [38].

We will not include these couplings here. Fermion mass terms can be built using the field $U=\Sigma_{1} \Sigma_{2} \cdots \Sigma_{K+1}$ transforming as $U \rightarrow L U R^{\dagger}$ [30]. The case $K=1$ corresponds to the BESS model [31, 32] whose phenomenology was studied at the LHC in Ref. [39]. This model has been recently rediscovered as a three site Higgsless model [40], and its phenomenology has now received a renewed attention [9, 33, 34, 35]. The requirement of consistency of the model with electroweak precision data can be satisfied only via a strong cancelation between gauge-mixing and $b$-parameter contributions to $\epsilon_{3}(S)$, ending up with fermiophobic vector resonances.

In this paper we concentrate on the case $K=2$ where the EWPT constraints can be satisfied without having necessarily fermiophobic resonances. The Drell-Yan channels become relevant here. For simplicity we will assume $g_{1}=g_{2}$ and $f_{1}=f_{3}$. This choice corresponds to a L-R symmetry in the new gauge sector, leading to a definite parity for the corresponding gauge bosons once the standard gauge interactions are turned off.

Summing up, the parameters of the model are $g_{1}, f_{1}, f_{2}, b_{1}$ and $b_{2}$. For the gauge sector, this model corresponds to the one proposed in Ref. [36] once fixed the set of parameters to be: $a=0, b=c=d / 2=2 f_{1}^{2} / v^{2}, g^{\prime \prime}=\sqrt{2} g_{1}, v=\left(\sqrt{2} G_{F}\right)^{-1 / 2}$. In Appendix A, we give the charged and neutral gauge bosons spectrum in the four site model.

\section{A. Fermion gauge boson couplings}

Using the explicit relations between gauge fields $\left(\tilde{W}, \tilde{A}^{1,2}\right)$ and mass eigenvectors $\left(W, W_{1,2}^{ \pm}\right)$given in Eqs. (A8 A10), we get the charged fermion-boson interaction:

$$
\mathcal{L}_{C C}=\bar{\psi}_{L} \gamma^{\mu} T^{-} \psi_{L}\left(a_{W} W_{\mu}^{+}+a_{1}^{c} W_{1 \mu}^{+}+a_{2}^{c} W_{2 \mu}^{+}\right)+\text {h.c. }
$$

with

$$
\begin{gathered}
a_{W}=-\frac{\tilde{g}}{\sqrt{2}}\left(1-\frac{b}{2}\right)\left(1-\frac{\tilde{g}^{2}}{g_{1}^{2}} \frac{z_{W}}{2}\right) \\
a_{1}^{c}=-\frac{g_{1}}{2\left(1+b_{+}\right)}\left(b_{+}-\frac{\tilde{g}^{2}}{g_{1}^{2}}\right) \\
a_{2}^{c}=-\frac{g_{1}}{2\left(1+b_{+}\right)}\left(b_{-}-\frac{\tilde{g}^{2}}{g_{1}^{2}} z^{2}\right)
\end{gathered}
$$


where

$$
z_{W}=\frac{1}{2}\left(1+z^{4}\right) \quad b=\frac{b_{+}-b_{-} z^{2}}{\left(1+b_{+}\right)} \quad b_{ \pm}=b_{1} \pm b_{2} \quad z=\frac{f_{1}}{\sqrt{f_{1}^{2}+2 f_{2}^{2}}}
$$

and we have neglected terms $\mathcal{O}\left(\tilde{g}^{4} / g_{1}^{4}\right)$ and $\mathcal{O}\left(b_{i} \tilde{g}^{2} / g_{1}^{2}\right)$. The reason why we neglect terms $\mathcal{O}\left(b_{i} \tilde{g}^{2} / g_{1}^{2}\right)$ is that the direct fermion couplings to the new resonances are of the order $b_{i} g_{1}$ and we do not expect couplings bigger than the SM ones to be allowed, that is $b_{i} \leq \tilde{g} / g_{1}$. This is confirmed by the bounds from LEP discussed hereafter. Notice also that $0 \leq z<1$ and, from Eq. (A7), $M_{1}=z M_{2}<M_{2}$.

In analogous way, using the relations between gauge fields $\left(\tilde{W}^{3}, \tilde{Y}, \tilde{A}_{1,2}^{3}\right)$ and neutral eigenvectors $\left(A, Z, Z_{1,2}\right)$ given in Eqs. (A18) $\left.A 21\right)$ we derive the neutral fermion-boson interactions:

$$
\mathcal{L}_{N C}=\bar{\psi} \gamma^{\mu}\left(-a_{F} \mathbf{Q} A_{\mu}+a_{1}^{n} Z_{1 \mu}+a_{2}^{n} Z_{2 \mu}+a_{Z} Z_{\mu}\right) \psi
$$

with

$$
\begin{gathered}
a_{F}=\tilde{g} s_{\tilde{\theta}}\left(1-\frac{\tilde{g}^{2}}{g_{1}^{2}} z_{\gamma}\right) \equiv e \\
a_{Z}=-\frac{\tilde{g}}{c_{\tilde{\theta}}}\left(1-\frac{b}{2}\right)\left(1-\frac{\tilde{g}^{2}}{g_{1}^{2}} \frac{z_{Z}}{2}\right)\left[\mathbf{T}^{3}-\frac{s_{\tilde{\theta}}^{2}}{\left(1-\frac{b}{2}\right)}\left(1-\frac{\tilde{g}^{2} c_{\tilde{\theta}}}{g_{1}^{2} s_{\tilde{\theta}}} z_{Z_{\gamma}}\right) \mathbf{Q}\right] \\
a_{1}^{n}=-\frac{g_{1}}{\sqrt{2}\left(1+b_{+}\right)}\left(b_{+}-\frac{\tilde{g}^{2}}{g_{1}^{2}} \frac{c_{2 \tilde{\theta}}}{c_{\tilde{\theta}}^{2}}\right) \mathbf{T}^{3}+\frac{\tilde{g}^{2} \tan ^{2} \tilde{\theta}}{\sqrt{2} g_{1}} \mathbf{Q} \\
a_{2}^{n}=-\frac{g_{1}}{\sqrt{2}\left(1+b_{+}\right)}\left(b_{-}-\frac{\tilde{g}^{2}}{g_{1}^{2}} \frac{z^{2}}{c_{\tilde{\theta}}^{2}}\right) \mathbf{T}^{3}-\frac{\tilde{g}^{2} z^{2} \tan ^{2} \tilde{\theta}}{\sqrt{2} g_{1}} \mathbf{Q}
\end{gathered}
$$

where

$$
z_{\gamma}=s_{\tilde{\theta}}^{2}, \quad z_{Z}=\frac{1}{2} \frac{\left(z^{4}+c_{2 \tilde{\theta}}^{2}\right)}{c_{\tilde{\theta}}^{2}}, \quad z_{Z \gamma}=-\tan \tilde{\theta} c_{2 \tilde{\theta}}
$$

and $\mathbf{T}^{3}=\tau_{L}^{3} / 2\left(\tau_{L}^{3} \psi_{L}= \pm \psi_{L}\right.$ and $\left.\tau_{L}^{3} \psi_{R}=0\right), \tan \tilde{\theta}=s_{\tilde{\theta}} / c_{\tilde{\theta}}=\tilde{g}^{\prime} / \tilde{g}, \mathbf{Q}$ is the electric charge in unit $e$ (the proton charge).

\section{BOUNDS FROM EWPT AND FROM PERTURBATIVE UNITARITY}

Simplest Higgsless models suffer for a tension between EWPT and perturbative unitarity. This tension can be alleviated by allowing for delocalization of fermions in the fifth dimension [7, 29] or by allowing for direct couplings of the new gauge bosons to standard matter [30]. We will explore this second possibility in the four site model. 


\section{A. Bounds from EWPT}

In order to get bounds on the parameter space of the model, it is convenient to derive the new physics contribution to the electroweak parameters $\epsilon_{1}, \epsilon_{2}$ and $\epsilon_{3}$ (or $S, T$ and $U$ ) which are strongly constrained by the electroweak measurements [41].

These parameters can be obtained from $\Delta r_{W}, \Delta \rho$ and $\Delta k$ [28, 42] :

$$
\begin{aligned}
\epsilon_{1} & =\Delta \rho \\
\epsilon_{2} & =c_{\theta}^{2} \Delta \rho+\frac{s_{\theta}^{2}}{c_{2 \theta}} \Delta r_{W}-2 s_{\theta}^{2} \Delta k \\
\epsilon_{3} & =c_{\theta}^{2} \Delta \rho+c_{2 \theta} \Delta k
\end{aligned}
$$

with $\Delta r_{W}$ defined by:

$$
\frac{M_{W}^{2}}{M_{Z}^{2}}=c_{\theta}^{2}\left[1-\frac{s_{\theta}^{2}}{c_{2 \theta}} \Delta r_{W}\right]
$$

$\Delta \rho$ and $\Delta k$ given in terms of the neutral current couplings to the $Z$ gauge boson

$$
\mathcal{L}^{\text {neutral }}(Z)=-\frac{e}{s_{\theta} c_{\theta}}\left(1+\frac{\Delta \rho}{2}\right) Z_{\mu} \bar{\psi}\left[\gamma^{\mu} g_{V}+\gamma^{\mu} \gamma_{5} g_{A}\right] \psi
$$

with

$$
g_{V}=\frac{\mathbf{T}^{\mathbf{3}}}{2}-s_{\theta_{e f f}}^{2} \mathbf{Q}, \quad g_{A}=-\frac{\mathbf{T}^{\mathbf{3}}}{2}, \quad s_{\theta_{e f f}}^{2}=(1+\Delta k) s_{\theta}^{2}
$$

and $s_{\theta}$ defined by:

$$
s_{\theta}^{2} c_{\theta}^{2}=\frac{\sqrt{2} e^{2}}{8 M_{Z}^{2} G_{F}} .
$$

Therefore, in this scheme, the physical inputs are chosen to be the electric charge, the Fermi constant and the $Z$-boson mass.

As already said, the parameters of the model are $g_{1}, f_{1}, f_{2}, b_{1}$ and $b_{2}$. Fixing $M_{Z}$, given in our model by Eq. (A14), to its experimental value, we get a relation among the five initial parameters which allows to express $g_{1}$ in terms of the others. This implies that the model has four independent free parameters. By Eq. (A7), we prefer to use the masses instead of the link couplings; we thus end up with $M_{1}, M_{2}, b_{1}$ and $b_{2}$ as free parameters. The explicit relation for $g_{1}$ is (at leading order):

$$
g_{1} \sim \frac{e}{s_{2 \theta}} \frac{M_{1}}{M_{Z}} \sqrt{2\left(1-z^{2}\right)}
$$

Let us compute the expression for the Fermi constant $G_{F}$ in our model. Neglecting terms $\mathcal{O}\left(\tilde{g}^{4} / g_{1}^{4}\right)$ and $\mathcal{O}\left(b_{i} \tilde{g}^{2} / g_{1}^{2}\right)$. We get

$$
\frac{G_{F}}{\sqrt{2}}=\frac{a_{W}^{2}}{4 M_{W}^{2}}+\frac{\left(a_{1}^{c}\right)^{2}}{4 M_{1, c}^{2}}+\frac{\left(a_{2}^{c}\right)^{2}}{4 M_{2, c}^{2}}=\frac{\tilde{g}^{2}}{8 M_{W}^{2}}\left(1-\frac{b}{2}\right)^{2}\left(1-\frac{\tilde{g}^{2}}{g_{1}^{2}} z_{W}\right)+\frac{\beta}{4}
$$


with

$$
\beta=\frac{\left(1-z^{2}\right)\left(b_{+}^{2}+b_{-}^{2} z^{2}\right)}{8\left(1+b_{+}\right)^{2} f^{2}}, \quad f=\frac{f_{1} f_{2}}{\sqrt{f_{1}^{2}+2 f_{2}^{2}}}
$$

and $M_{W}, M_{1, c}, M_{2, c}$, given in Eqs. (A3) As ). Using also Eq. (A14) we get the relation between $M_{W}$ and $M_{Z}$

$$
M_{W}^{2}=M_{Z}^{2} c_{\tilde{\theta}}^{2}\left(1-\frac{e^{2}}{g_{1}^{2} s_{\tilde{\theta}}^{2}}\left(z_{W}-z_{Z}\right)\right)
$$

where we have used Eq. (11), again neglecting terms $\mathcal{O}\left(\tilde{g}^{4} / g_{1}^{4}\right)$ and $\mathcal{O}\left(b_{i} \tilde{g}^{2} / g_{1}^{2}\right)$. Requiring the tree-level SM relation in Eq. (20) to hold true also in our model, we derive the relation between the $\tilde{\theta}$ angle and the physical inputs:

$$
s_{\tilde{\theta}}^{2}=\frac{1}{2}\left(1-\sqrt{1-s_{2 \theta}^{2} X}\right)
$$

where

$$
X=\frac{1}{R}\left[1+\frac{4 e^{2}}{g_{1}^{2}}\left(\frac{R z_{W}}{s_{2 \theta}^{2}}-1\right)\right], \quad R=\frac{1-\frac{\beta}{2 \sqrt{2} G_{F}}}{\left(1-\frac{b}{2}\right)^{2}}
$$

By using the definitions of $\Delta \rho, \Delta r_{W}, \Delta k$ and Eqs. (22, 24, 25) we get, again neglecting terms $\mathcal{O}\left(\tilde{g}^{4} / g_{1}^{4}\right)$ and $\mathcal{O}\left(\tilde{g}^{2} / g_{1}^{2} b_{i}\right)$

$$
\begin{gathered}
\Delta \rho=2\left(-1+\sqrt{1-\frac{\beta}{2 \sqrt{2} G_{F}}}\right) \\
\Delta k=-1+\frac{s_{\tilde{\theta}}^{2}}{s_{\theta}^{2}} \frac{1}{\left(1-\frac{b}{2}\right)}\left[1-\frac{e^{2}}{g_{1}^{2} s_{\tilde{\theta}}^{2}} z_{Z \gamma}\right] \\
\Delta r_{W}=\frac{c_{2 \theta}}{s_{\theta}^{2}}\left[1-\frac{c_{\tilde{\theta}}^{2}}{c_{\theta}^{2}}\left(1-\frac{e^{2}}{g_{1}^{2} s_{\tilde{\theta}}^{2}}\left(z_{W}-z_{Z}\right)\right)\right]
\end{gathered}
$$

We can now compute the new physics contribution to the $\epsilon_{1,2,3}$ by using Eq. (16) as in Ref. [30]. We find:

$$
\epsilon_{1,2}=-\frac{\left(1-z^{2}\right)\left(b_{+}^{2}+z^{2} b_{-}^{2}\right)}{4}, \quad \epsilon_{3}=\frac{g^{2}}{2 g_{1}^{2}}\left(1-z^{4}\right)-\frac{b}{2}
$$

with $b$ and $b_{ \pm}$given in Eq. (9) and $g=e / s_{\theta}$. As previously mentioned, while $\epsilon_{3}$ is strongly affected by the direct fermion-boson couplings, having a linear dependence on the $b$-parameter, $\epsilon_{1}$ and $\epsilon_{2}$ receive a mild contribution. They display in fact only a quadratic behaviour in $b_{ \pm}$, owing to the $S U(2)$ custodial symmetry.

In order to get bounds on the model parameters we have to compare with the experimental values for the $\epsilon_{1,2,3}[41]$ :

$$
\epsilon_{1}^{e x p}=(5.0 \pm 1.1) \times 10^{-3}, \quad \epsilon_{2}^{e x p}=(-8.8 \pm 1.2) \times 10^{-3}, \quad \epsilon_{3}^{e x p}=(4.8 \pm 1.0) \times 10^{-3}
$$


after adding to the new physics contribution the radiative corrections. In our analysis, we have only considered the SM radiative corrections evaluated for $m_{t}=172.5 \mathrm{GeV}$ and an heavy Higgs boson, $m_{H}=1 \mathrm{TeV}$, taken as a cut-off:

$$
\epsilon_{1}^{r a d}=3.4 \times 10^{-3}, \quad \epsilon_{2}^{r a d}=-6.5 \times 10^{-3}, \quad \epsilon_{3}^{r a d}=6.7 \times 10^{-3} .
$$

In Figs. 1 and 2, we show the 95\% C.L. experimental bounds from $\epsilon_{1}$ and $\epsilon_{3}$ ( $\epsilon_{2}$ doesn't give any relevant limit thanks to its negative experimental value) in the plane $\left(b_{1}, b_{2}\right)$ for different choices of heavy gauge boson masses, $M_{1,2}$, at fixed ratio $z=M_{1} / M_{2}$ (see Eq. A7). The bounds are obtained by the standard $\chi$-square fit

$$
\chi_{i}^{2}=\left(\frac{\epsilon_{i}+\epsilon_{i}^{\text {rad }}-\epsilon_{i}^{\text {exp }}}{\sigma_{i}^{\text {exp }}}\right)^{2}=5.99 \quad \text { with } i=1,3
$$

which has been calculated exactly in the $b_{i}$ parameters. As an example of the dependence of the $\epsilon_{i}$ constraints on the free parameter $z$, we consider four values $z=(0.2,0.4,1 / \sqrt{3}, 0.8)$ and show the corresponding results in the four plots of Figs. 1 and 2. From there, it is clear that for $z=0.2$ there is a little match between experimental data and model parameters, instead for $z \geq 0.4$ there is a non negligible allowed strip. For each choice of $z$ we choose $M_{1,2}$ to vary inside the region allowed by the perturbative unitarity limit discussed in the second part of this section. The bounds from $\epsilon_{1}$ are quite insensitive to the value of the resonance masses, those from $\epsilon_{3}$ goes from up to down by increasing $M_{1}$. Also, the range of low $M_{1}$ values for given $z$ has been cut in order to be consistent with our neglecting of terms of $O\left(\tilde{g}^{4} / g_{1}^{4}\right)$ and taking into account Eq. (21).

It is instructive to translate these bounds on the plane delimited by the direct couplings between the two charged (neutral) gauge bosons, $W_{1,2}^{ \pm}\left(Z_{1,2}\right)$, and ordinary matter. In fact, the following expression for $\epsilon_{3}$ can be easily derived by using Eqs. (7), (8), (13), (14) and neglecting terms $\mathcal{O}\left(\tilde{g}^{4} / g_{1}^{4}\right)$ and $\mathcal{O}\left(b_{i} \tilde{g}^{2} / g_{1}^{2}\right)$ :

$$
\epsilon_{3} \sim \frac{1}{g_{1}}\left(a_{1}^{c}-z^{2} a_{2}^{c}\right) \sim-\frac{\sqrt{2}}{g_{1}}\left(a_{1 L}^{e}-z^{2} a_{2 L}^{e}\right)-\frac{e^{2}}{g_{1}^{2} c_{\theta}^{2}}\left(1+z^{4}\right)
$$

where $a_{1,2}^{c}$ are the fermion couplings of the charged $W_{1,2}^{ \pm}$-boson and $a_{i L}^{e}(i=1,2)$ are the couplings of the neutral $Z_{1,2}$-boson to the left-handed electron component. ¿From the previous relations we note that the contribution of the fermion couplings of $Z_{2}$ and $W_{2}^{ \pm}$are multiplied by a factor $z^{2}$. As a consequence, in order to satisfy the stringent bounds from $\epsilon_{3}$, fermion couplings of the $Z_{1}$ and $W_{1}^{ \pm}$resonances will be much more constrained with respect to the 
$Z_{2}$ and $W_{2}^{ \pm}$ones.
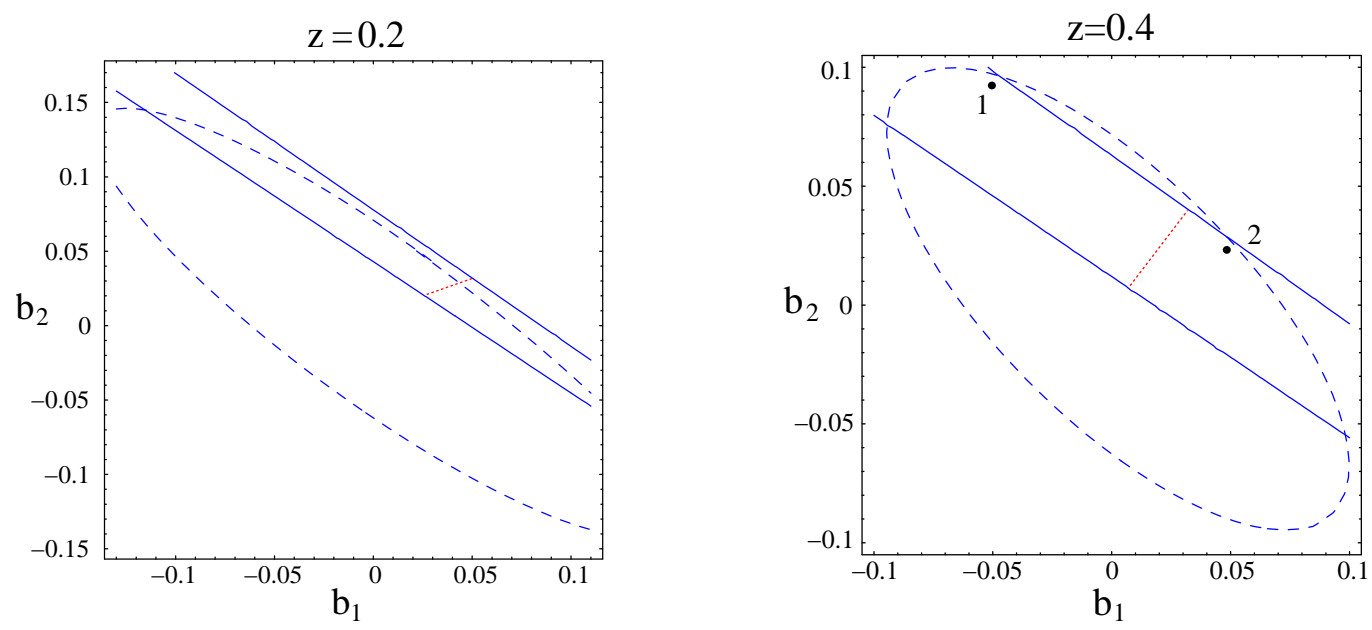

FIG. 1: $95 \%$ C.L. bounds on the plane $\left(b_{1}, b_{2}\right)$ from $\epsilon_{1}$ (dash line) and $\epsilon_{3}$ (solid line). Left panel: $z=0.2$ and $450 \leq M_{1}(\mathrm{GeV}) \leq 550$. Right panel: $z=0.4,500 \leq M_{1}(\mathrm{GeV}) \leq 1000$. The allowed regions are the internal ones. The red dots represent the so-called ideal cancellation. Also shown are the points corresponding to the scenarios 1 and 2 of the following phenomenological analysis.
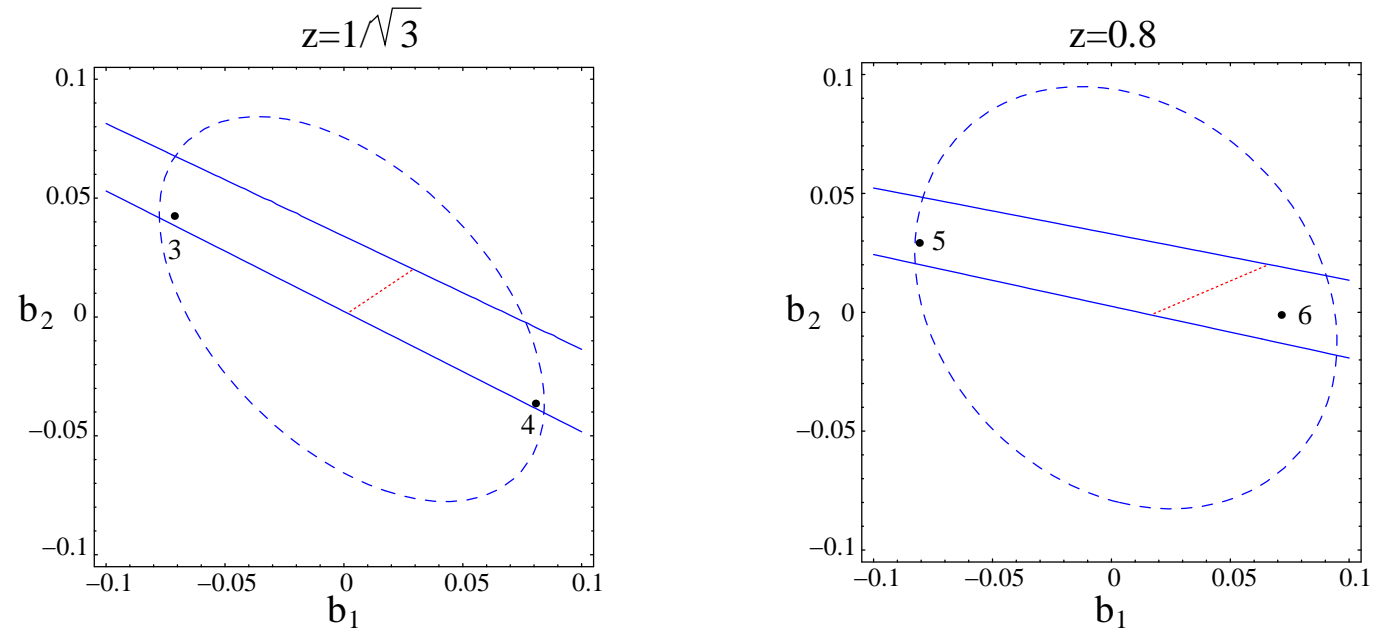

FIG. 2: Same as Fig 1. Left panel: $z=1 / \sqrt{3}$ (corresponding to $f_{1}=f_{2}$ ), $700 \leq M_{1}(\mathrm{GeV}) \leq 1750$. Right panel: $z=0.8,700 \leq M_{1}(\mathrm{GeV}) \leq 1600$. Also shown are the points corresponding to the scenarios $3,4,5$ and 6 of the following phenomenological analysis.

Let us derive the experimental bounds from $\epsilon_{1}$ and $\epsilon_{3}$ for the charged and neutral fermion couplings normalized with respect to the SM ones. The results are shown in Fig. 3 for 

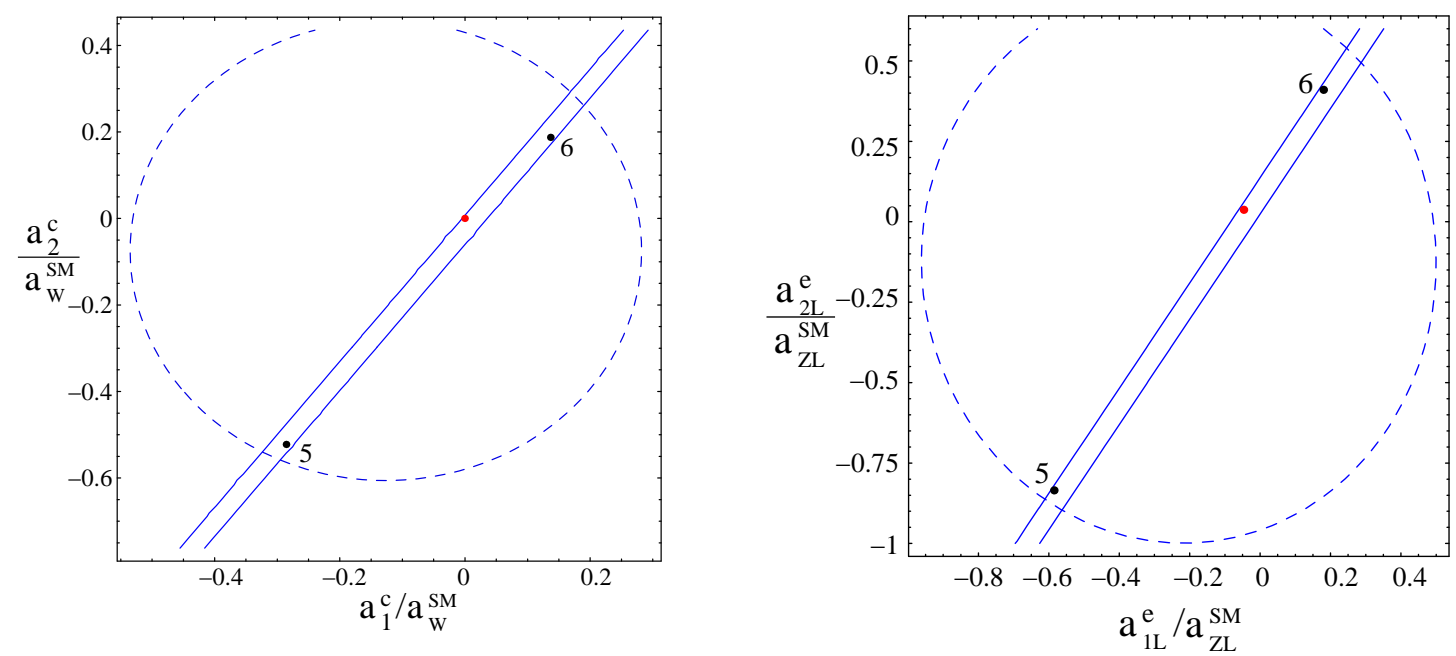

FIG. 3: $95 \%$ C.L. bounds from $\epsilon_{1}$ (dashed line) and $\epsilon_{3}$ (solid line), for $M_{1}=1 \mathrm{TeV}$ and $z=0.8$ on the plane $\left(a_{1}^{c} / a_{W}^{S M}, a_{2}^{c} / a_{W}^{S M}\right)$ with $a_{W}^{S M}=-e /\left(\sqrt{2} s_{\theta}\right)$ (left panel), on the plane $\left(a_{1 L}^{e} / a_{Z L}^{S M}, a_{2 L}^{e} / a_{Z L}^{S M}\right)$ with $a_{Z L}^{S M}=-e /\left(s_{\theta} c_{\theta}\right)\left(1 / 2-s_{\theta}^{2}\right)$ (right panel). In both cases the central dot represents the socalled ideal cancellation. Also shown are the points corresponding to the scenarios 5 and 6 of the following phenomenological analysis.

$M_{1}=1 \mathrm{TeV}$ and $z=0.8$. The measurement of $\epsilon_{3}$ strongly constraints the physical space of the couplings, instead the measurement of $\epsilon_{1}$ gives looser bounds. The central dot represents the point where $a_{1}^{c}=a_{2}^{c}=0$, the so-called ideal cancellation [30, 38, 43, 44, 45]. This is the assumption under which the most recent phenomenological analysis have been performed at the LHC within the three site Higgsless model [34, 35]. There, in fact, the new resonances are forced by EWPT to be fermiophobic (or almost fermiophobic). In the four site extension instead, while the relation between the couplings of the two gauge-boson triplets with SMfermions is strongly constrained by the $\epsilon_{3}$-parameter, their magnitude is weakly limited by $\epsilon_{1}$. As a result, the direct fermion-boson couplings can be of the same order of the SM ones, as shown in Fig. 3. The phenomenological consequence is that, while the minimal Higgsless model can be explored only in very complex multi-particle processes (triple gauge boson production or vector boson fusion channels) which require high luminosity, the four site Higgsless model could be proved in the more promising Drell-Yan channel already at the LHC start-up.

We are not considering in this paper new physics loop corrections to the $\epsilon$ parameters: 
it has been recently shown that in some Higgsless model this effect can be important for reconciling these schemes with electroweak precision data [46, 47, 48].

We have instead considered the limits from LEP2 by computing the $\hat{S}, \hat{T}, \hat{U}, W, Y, V$ and $\mathrm{X}$ [41]. The result is that $\hat{T}, \hat{U}$, and $V$ vanish due to the $\mathrm{SU}(2)$ custodial symmetry, $X, Y$ and $W$ are of order $b_{i} \tilde{g}^{2} / g_{1}^{2}$ or $\tilde{g}^{4} / g_{1}^{4}$ and therefore do not alter the stringent limits already obtained from $\epsilon_{3}$.

\section{B. Perturbative unitarity bounds}

As well known, in the SM without a light Higgs the vector boson scattering (VBS) amplitudes violate perturbative unitarity at an energy scale of the order of $\Lambda_{\mathrm{HSM}} \simeq 1.7 \mathrm{TeV}$ [49]. One of the strong motivations for Higgsless models, derived from 5D local gauge theories, is their ability to delay such a unitarity violation to higher energies, via the exchange of the predicted extra gauge bosons.

In this section, we briefly discuss the issue of partial wave unitarity. The corresponding bound can be easily obtained by using the Equivalence Theorem relating, at high energy, the gauge boson scattering amplitudes to the corresponding Goldstone ones [49, 50, 51, 52]. Let us put $\tilde{g}=\tilde{g}^{\prime}=0$ and $K=2$ in the Lagrangian given in Eq. (2) and expand the fields as $\Sigma_{i}=\exp \left[i \alpha_{i} \vec{\pi}_{i} \cdot \vec{\tau}\right]$. We get:

$$
\begin{aligned}
\sum_{i=1}^{3} f_{i}^{2} \operatorname{Tr}\left[D_{\mu} \Sigma_{i}^{\dagger} D^{\mu} \Sigma_{i}\right] \sim & \sum_{i=1}^{3}\left[2 f_{i}^{2} \alpha_{i}^{2} \partial_{\mu} \vec{\pi}_{i} \cdot \partial^{\mu} \vec{\pi}_{i}+\frac{2}{3} \alpha_{i}^{4} f_{i}^{2}\left[\left(\vec{\pi}_{i} \cdot \partial_{\mu} \vec{\pi}_{i}\right)^{2}-\vec{\pi}_{i}^{2}\left(\partial_{\mu} \vec{\pi}_{i}\right)^{2}\right]\right] \\
& +\sum_{i=1}^{2} g_{i} \overrightarrow{\tilde{A}}_{i}^{\mu} \cdot\left(f_{i}^{2} \alpha_{i} \partial_{\mu} \vec{\pi}_{i}-f_{i+1}^{2} \alpha_{i+1} \partial_{\mu} \vec{\pi}_{i+1}\right)+\cdots
\end{aligned}
$$

The unitary gauge for the $\tilde{A}_{i}$ gauge bosons is given by

$$
\alpha_{i}=\frac{f}{2 f_{i}^{2}}, \quad \vec{\pi}_{i}(x)=\vec{\pi}(x)
$$

with $f$ given in Eq. (23), which ensures a canonical kinetic term for the $\pi$ 's:

$$
\sum_{i=1}^{3} 2 f_{i}^{2} \alpha_{i}^{2} \partial_{\mu} \vec{\pi}_{i} \cdot \partial^{\mu} \vec{\pi}_{i}=\frac{1}{2} \partial_{\mu} \vec{\pi} \cdot \partial^{\mu} \vec{\pi} .
$$

We can now easily compute the scattering amplitude $\mathcal{A}_{W_{L}^{+} W_{L}^{-} \rightarrow W_{L}^{+} W_{L}^{-}}$which, for $\sqrt{s}>>M_{W}$, is equal to $\mathcal{A}_{\pi^{+} \pi^{-} \rightarrow \pi^{+} \pi^{-}}$due to the Equivalence Theorem. This amplitude for high energy 
$\left(\sqrt{s}>>M_{1,2}\right)$ is dominated by the four-pion vertex which can be extracted from Eq. (344). Comparing the four-linear Lagrangian coefficient in Eq. (34), computed in unitary gauge, with the analogous interaction given in the SM without a light Higgs, we get

$$
\frac{1}{6 v_{S M}^{2}}=\frac{f^{4}}{24} \sum_{i=1}^{3} \frac{1}{f_{i}^{6}} .
$$

The pion-pion scattering amplitudes can thus be written in our model as:

$$
\mathcal{A}_{\pi^{+} \pi^{-} \rightarrow \pi^{+} \pi^{-}} \sim-\frac{f^{4}}{4} \sum_{i=1}^{3} \frac{u}{f_{i}^{6}}
$$

where $u$ is the Mandelstam variable, $u=-s(1-\cos \theta) / 2$, with $s$ the CM-energy squared and $\theta$ the $\pi^{-}$scattering angle. We also get:

$$
\mathcal{A}_{\pi^{3} \pi^{3} \rightarrow \pi^{3} \pi^{3}} \sim 0, \quad \mathcal{A}_{\pi^{+} \pi^{-} \rightarrow \pi^{3} \pi^{3}} \sim \frac{f^{4}}{4} \sum_{i=1}^{3} \frac{s}{f_{i}^{6}}
$$

which are related to the physical scattering amplitudes $Z_{L} Z_{L} \rightarrow Z_{L} Z_{L}$ and $W_{L}^{+} W_{L}^{-} \rightarrow Z_{L} Z_{L}$, respectively.

By considering the zero-isospin partial wave matrix for all VBS amplitudes with SM longitudinal gauge bosons as external states

$$
a_{0}=\frac{1}{16 \pi} \frac{s}{v_{S M}^{2}}\left(\begin{array}{cc}
\frac{1}{2} & \frac{1}{\sqrt{2}} \\
\frac{1}{\sqrt{2}} & 0
\end{array}\right)
$$

with normalization factors for the two channels $W_{L}^{+} W_{L}^{-}$and $(1 / \sqrt{2}) Z_{L} Z_{L}$ as in Ref. [49], and requiring the partial wave bound $\left|a_{0}\right|=1$ for the maximum eigenvalue, we get the result shown by the green (lighter) curve in Fig. 4. The maximum energy scale, up to which the perturbative unitarity can be delayed, is reached for $z=1 / \sqrt{3}$ or equivalently $f_{1}=f_{2}$ which can be interpreted as a flat-metric scenario. In this particular case, such a delay with respect to the answer of the SM without a light Higgs is modulated by the factor $(K+1) \equiv 3$ : $\Lambda_{\text {four-site }}=3 \Lambda_{\mathrm{HSM}} \sim 5 \mathrm{TeV}$. However, the four site model has in addition two vector-boson triplets with, potentially, bad behaving longitudinal scattering amplitudes. In order to be predictive, one has to require a fully perturbative regime for all involved particles. The unitarity limit must thus be extended, in order to ensure a good high energy behaviour for all scattering amplitudes, i.e. with both SM and extra gauge bosons as external states.

For energies much higher than the masses of the new vector bosons $\left(\sqrt{s}>>M_{1,2}\right)$, we can determine such a unitarity bound by considering the eigenchannel amplitudes corresponding 


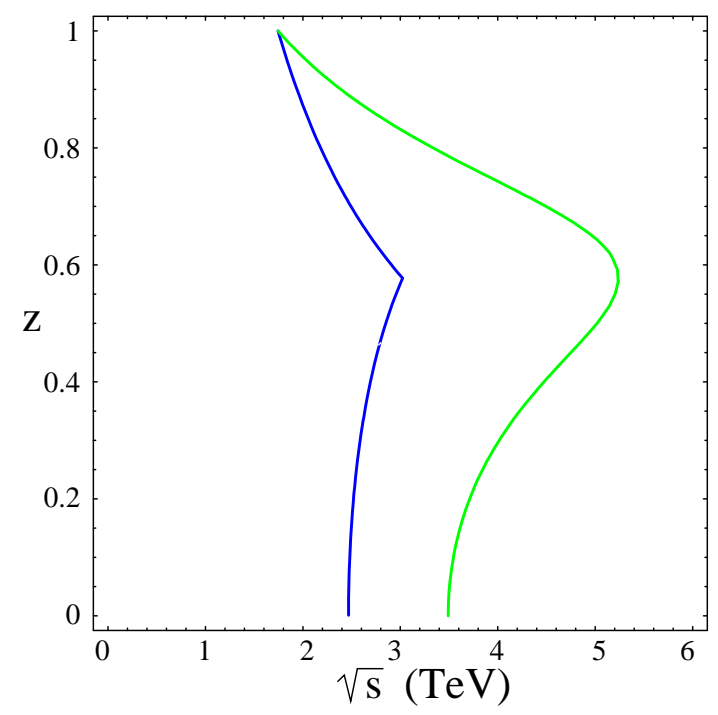

FIG. 4: Partial wave unitarity bounds on the plane $(\sqrt{s}, z)$, by requiring $\left|a_{0}\right| \leq 1$ for scattering amplitudes with external SM gauge bosons (green lighter curve), and for scattering amplitudes with both SM and extra gauge bosons as external states (blue darker curve). The allowed regions are on the left of the curves.

to all possible longitudinal vector bosons. This can be done again by using the Equivalence Theorem and evaluating the chiral Lagrangian in the unitary gauge for all the vector bosons given by $\Sigma_{i}=\exp \left[i \vec{\pi}_{i} \cdot \vec{\tau} /\left(2 f_{i}\right)\right]$. In this gauge, the amplitudes are diagonal and the high energy result is simply given by:

$$
\mathcal{A}_{\pi_{i}^{+} \pi_{i}^{-} \rightarrow \pi_{i}^{+} \pi_{i}^{-}} \sim-\frac{u}{4 f_{i}^{2}}, \quad \mathcal{A}_{\pi_{i}^{3} \pi_{i}^{3} \rightarrow \pi_{i}^{3} \pi_{i}^{3}} \sim 0, \quad \mathcal{A}_{\pi_{i}^{+} \pi_{i}^{-} \rightarrow \pi_{i}^{3} \pi_{i}^{3}} \sim \frac{s}{4 f_{i}^{2}}
$$

Requiring $\left|a_{0}\right|=1$ for the maximum eigenvalue, we get the blue (darker) curve shown in Fig. 4. We see that, also in this case, the most delayed unitarity limit is realized for the choice $f_{1}=f_{2}$ that is $z=1 / \sqrt{3}$, and corresponds to the rescaling $\Lambda_{\text {four-site }}^{\text {total }}=\sqrt{K+1} \Lambda_{\mathrm{HSM}} \sim$ $3 \mathrm{TeV}$. The four site Higgsless model can thus preserve perturbative unitarity over practically the whole effective energy range of the LHC. The spectrum of the new predicted particles $W_{1,2}^{ \pm}$and $Z_{1,2}$ must lie within that region: $M_{1,2} \leq \Lambda_{\text {four-site }}^{\text {total }}$

In our following phenomenological analysis, the most stringent unitarity bound in Fig. 4 is considered. We conclude by noting that the three site unitarity limits are lower or equal to the ones of the four site model, given by the blue (darker) line of Fig. 4, depending on the value of the ratio $f_{1} / \sqrt{f_{1}^{2}+f_{2}^{2}}$. 


\section{DRELL-YAN PRODUCTION AT THE LHC}

We can now consider the production of the six new gauge bosons, $W_{1,2}^{ \pm}$and $Z_{1,2}$, predicted by the four site Higgsless model at the LHC through Drell-Yan channels. Owing to the introduction of direct couplings between ordinary matter and extra gauge bosons, in addition to the usual indirect ones due to the mixing, the experimental bounds from electroweak precision data on the model parameters are indeed less stringent. As a consequence, and in contrast with the existing fermiophobic Higgsless literature, quite large couplings between SM fermions and extra gauge bosons are allowed (see Fig. 3).

\section{A. Processes and their computation}

We analyze in detail two classes of processes,

(i) $\mathrm{pp} \rightarrow l^{+} l^{-}$, with $l=\mathrm{e}, \mu, \quad$ and

(ii) $\mathrm{pp} \rightarrow l \nu_{l}$, with $l=\mathrm{e}, \mu$.

The first class is characterized by two isolated charged leptons in the final state. The latter gives instead rise to one isolated charged lepton plus missing energy. In our notation, $l \nu_{l}$ indicates both $l^{-} \bar{\nu}_{l}$ and $l^{+} \nu_{l}$. The aforementioned neutral and charged Drell-Yan channels can involve the production of two neutral extra gauge bosons, $Z_{1}$ and $Z_{2}$, and four charged extra gauge bosons $W_{1}^{ \pm}$and $W_{2}^{ \pm}$as intermediate states, respectively. Both classes of processes are described by the formula

$$
\mathrm{d} \sigma^{h_{1} h_{2}}\left(P_{1}, P_{2}, p_{f}\right)=\sum_{i, j} \int \mathrm{d} x_{1} \mathrm{~d} x_{2} f_{i, h_{1}}\left(x_{1}, Q^{2}\right) f_{j, h_{2}}\left(x_{2}, Q^{2}\right) \mathrm{d} \hat{\sigma}^{i j}\left(x_{1} P_{1}, x_{2} P_{2}, p_{f}\right),
$$

where $p_{f}$ summarizes the final-state momenta, $f_{i, h_{1}}$ and $f_{j, h_{2}}$ are the distribution functions of the partons $i$ and $j$ in the incoming hadrons $h_{1}$ and $h_{2}$ with momenta $P_{1}$ and $P_{2}$, respectively, $Q$ is the factorization scale, and $\hat{\sigma}^{i j}$ represent the cross sections for the partonic processes. Since the two incoming hadrons are protons and we sum over final states with opposite charges, we find

$$
\begin{aligned}
\mathrm{d} \sigma^{h_{1} h_{2}}\left(P_{1}, P_{2}, p_{f}\right)=\int \mathrm{d} x_{1} \mathrm{~d} x_{2} & \sum_{Q=u, c, d, s, b}\left[f_{\overline{\mathrm{Q}}, \mathrm{p}}\left(x_{1}, Q^{2}\right) f_{\mathrm{Q}, \mathrm{p}}\left(x_{2}, Q^{2}\right) \mathrm{d} \hat{\sigma}^{\overline{\mathrm{Q}}}\left(x_{1} P_{1}, x_{2} P_{2}, p_{f}\right)\right. \\
& \left.+f_{\mathrm{Q}, \mathrm{p}}\left(x_{1}, Q^{2}\right) f_{\overline{\mathrm{Q}}, \mathrm{p}}\left(x_{2}, Q^{2}\right) \mathrm{d} \hat{\sigma}^{\mathrm{Q} \overline{\mathrm{Q}}}\left(x_{1} P_{1}, x_{2} P_{2}, p_{f}\right)\right]
\end{aligned}
$$


and

$$
\begin{aligned}
& \mathrm{d} \sigma^{h_{1} h_{2}}\left(P_{1}, P_{2}, p_{f}\right)=\int \mathrm{d} x_{1} \mathrm{~d} x_{2} \sum_{U=u, c} \sum_{D=d, s}\left[f_{\overline{\mathrm{D}}, \mathrm{p}}\left(x_{1}, Q^{2}\right) f_{\mathrm{U}, \mathrm{p}}\left(x_{2}, Q^{2}\right) \mathrm{d} \hat{\sigma}^{\overline{\mathrm{DU}}}\left(x_{1} P_{1}, x_{2} P_{2}, p_{f}\right)\right. \\
&+f_{\overline{\mathrm{U}}, \mathrm{p}}\left(x_{1}, Q^{2}\right) f_{\mathrm{D}, \mathrm{p}}\left(x_{2}, Q^{2}\right) \mathrm{d} \hat{\sigma}^{\overline{\mathrm{U}}}\left(x_{1} P_{1}, x_{2} P_{2}, p_{f}\right) \\
&+f_{\mathrm{D}, \mathrm{p}}\left(x_{1}, Q^{2}\right) f_{\overline{\mathrm{U}}, \mathrm{p}}\left(x_{2}, Q^{2}\right) \mathrm{d} \hat{\sigma}^{\mathrm{D} \overline{\mathrm{U}}}\left(x_{1} P_{1}, x_{2} P_{2}, p_{f}\right) \\
&\left.+f_{\mathrm{U}, \mathrm{p}}\left(x_{1}, Q^{2}\right) f_{\overline{\mathrm{D}}, \mathrm{p}}\left(x_{2}, Q^{2}\right) \mathrm{d} \hat{\sigma}^{\mathrm{U} \overline{\mathrm{D}}}\left(x_{1} P_{1}, x_{2} P_{2}, p_{f}\right)\right]
\end{aligned}
$$

for neutral and charged processes, respectively.

The tree-level amplitudes for the partonic processes have been generated by means of PHACT [53], a set of routines based on the helicity-amplitude formalism of Ref. [54]. The matrix elements have been inserted in the Monte Carlo event generator (MCEG) FAST_2f, dedicated to Drell-Yan processes at the EW and QCD leading order. FAST_2f can compute simultaneously the new-physics signal and the SM background. It can generate cross-sections and distributions for any observable, including any kind of kinematical cuts. The code is moreover interfaced with PYTHIA [55]. This feature can allow more realistic analysis, once FAST_2f is matched with detector simulation programs. This extension and relative study will be performed soon.

\section{B. Numerical setup}

For the numerical results presented here, we have used the following input values [37]: $M_{Z}=91.187 \mathrm{GeV}, \Gamma_{\mathrm{Z}}=2.512 \mathrm{GeV}, \Gamma_{\mathrm{W}}=2.105 \mathrm{GeV}, \alpha\left(M_{Z}\right)=1 / 128.88, G_{F}=1.166 \times$ $10^{-5} \mathrm{GeV}^{-2}$. Additional input parameters are the quark-mixing matrix elements [56], whose values have been taken to be $\left|V_{\mathrm{ud}}\right|=\left|V_{\mathrm{cs}}\right|=0.975,\left|V_{\mathrm{us}}\right|=\left|V_{\mathrm{cd}}\right|=0.222$, and zero for all other relevant matrix elements. In our scheme, the weak mixing-angle and the $W$-boson mass are derived quantities. For the matrix element evaluation, we adopt the fixed-width scheme. And, we use the CTEQ6L [57] for the parton distribution functions at the factorization scales:

$$
Q^{2}=M_{\mathrm{inv}}^{2}\left(l^{+} l^{-}\right)
$$

and

$$
Q^{2}=\frac{1}{2}\left(P_{\mathrm{T}}^{2}(l)+P_{\mathrm{T}}^{2}\left(\nu_{l}\right)\right)
$$


for neutral and charged Drell-Yan processes, respectively, where $M_{\mathrm{inv}}$ denotes the invariant mass and $P_{\mathrm{T}}$ is the transverse momentum. This scale choice appears to be appropriate for the calculation of differential cross sections, in particular for lepton distributions at high energy scales.

We have, moreover, implemented a general set of acceptance cuts, appropriate for LHC analysis, defined as follows:

- lepton transverse momentum $P_{\mathrm{T}}(l)>20 \mathrm{GeV}$,

- missing transverse momentum $P_{\mathrm{T}}^{\mathrm{miss}}>20 \mathrm{GeV}$ for $\mathrm{pp} \rightarrow l \nu_{l}$,

- lepton pseudo-rapidity $\left|\eta_{l}\right|<2.5$, where $\eta_{l}=-\log \left(\tan \theta_{l} / 2\right)$, and $\theta_{l}$ is the polar angle of the charged lepton $l$ with respect to the beam.

For the different processes considered, we have also used further cuts which are described in due time. We present results for the LHC at CM energy $\sqrt{s}=14 \mathrm{TeV}$ and an integrated luminosity from $L=100 \mathrm{pb}^{-1}$ to $L=100 \mathrm{fb}^{-1}$.

\section{Extra gauge boson production in Drell-Yan channels}

In the following two subsections we analyze the production of charged and neutral extra gauge bosons in Drell-Yan channels. We consider three different choices of mass spectrum and two sets of couplings to fermions for each choice inside the region allowed by EWPT and unitarity bounds:

$z=0.4$

\begin{tabular}{|c|c|c|c|}
\hline & $M_{1,2}(\mathrm{GeV})$ & $b_{1,2}$ & $g_{1}$ \\
\hline \hline 1 & 500,1250 & $-0.05,0.09$ & 2.7 \\
\hline 2 & 500,1250 & $0.06,0.02$ & 2.7 \\
\hline
\end{tabular}

$z=1 / \sqrt{3}$

\begin{tabular}{|c|c|c|c|}
\hline & $M_{1,2}(\mathrm{GeV})$ & $b_{1,2}$ & $g_{1}$ \\
\hline \hline 3 & 1732,3000 & $-0.07,0.04$ & 8.1 \\
\hline 4 & 1732,3000 & $0.08,-0.04$ & 8.1 \\
\hline
\end{tabular}

$z=0.8$

\begin{tabular}{|c|c|c|c|}
\hline & $M_{1,2}(\mathrm{GeV})$ & $b_{1,2}$ & $g_{1}$ \\
\hline \hline 5 & 1000,1250 & $-0.08,0.03$ & 3.5 \\
\hline 6 & 1000,1250 & $0.07,0.0$ & 3.5 \\
\hline
\end{tabular}

TABLE I: Choices of parameters for the six considered scenarios. The corresponding value of $g_{1}$ evaluated by Eq. (21) is also given.

These six examples give an idea of the possible scenarios predicted by the four site Higgsless model. In the model in fact the ratio between the gauge boson masses of the 
first and second triplet, i.e. $z=M_{1} / M_{2}$, is a free parameter. Hence, the distance between the two masses is arbitrary as well (actually the mass eigenvalues are slightly different from $M_{1,2}$ due to corrections $\mathcal{O}\left(\tilde{g} / g_{1}\right)^{2}$ as given in Appendix $\mathrm{A}$ and $\mathrm{B}$ ). We have thus chosen three cases, corresponding to $z=(0.4,1 / \sqrt{3}, 0.8)$, and representing from left to right very distant resonances, the flat-metric scenario, and a spectrum which tends to degeneracy by increasing $z$.

Also the magnitude of the couplings has a wide spectrum, as can be seen for example in Fig. 3 for the case $z=0.8$ and $M_{1}=1 \mathrm{TeV}$. In order to show the importance and the impact at the LHC of the related direct fermion-boson couplings, we consider two points in each plane $\left(b_{1}, b_{2}\right)$ : the first in the region down on the left of the allowed range, and the second high on right (see Figs. 1, 2). These are reported in the six scenarios listed above.

We are now ready to discuss numerical results for the charged and neutral Drell-Yan channels.

\section{1. $Z_{1}$ and $Z_{2}$ production at the $L H C$}

In this section, we present some cross sections and distributions for the leptonic process $\mathrm{pp} \rightarrow l^{+} l^{-}$with $l=\mathrm{e}, \mu$. These final states allow to analyze the production of the two neutral extra gauge bosons, $Z_{1,2}$, predicted by the four site Higgsless model.

General studies of Higgsless models have shown that the new strongly interacting vector bosons are expected to be produced at high CM energies. We thus select this kinematical configuration by imposing an additional cut on the invariant mass of the lepton pair, i.e. $M_{\text {inv }}\left(l^{+} l^{-}\right) \geq 400 \mathrm{GeV}$.

As an illustration of the behaviour and the impact of the new predicted particles at the LHC, we have chosen to analyze the following three differential cross-sections:

(i) distribution in the invariant mass of the reconstructed $Z_{1,2}$-boson, $M_{\mathrm{inv}}\left(l^{+} l^{-}\right)$,

(ii) distribution of the scattering angle in the $\mathrm{CM}$ frame, $\cos \theta_{l}^{*}$, taken in the region under the peak of the new resonances, i.e. $\left|M_{\text {inv }}\left(l^{+} l^{-}\right)-M_{i}\right| \leq 3 \Gamma_{i}(i=1,2)$,

(iii) forward-backward charge asymmetry versus the dilepton invariant mass.

As mentioned in Sect. IVC, we show results for three values of the $z$-parameter, $z=$ 
(a) $M_{1,2}=(500,1250) \mathrm{GeV}$

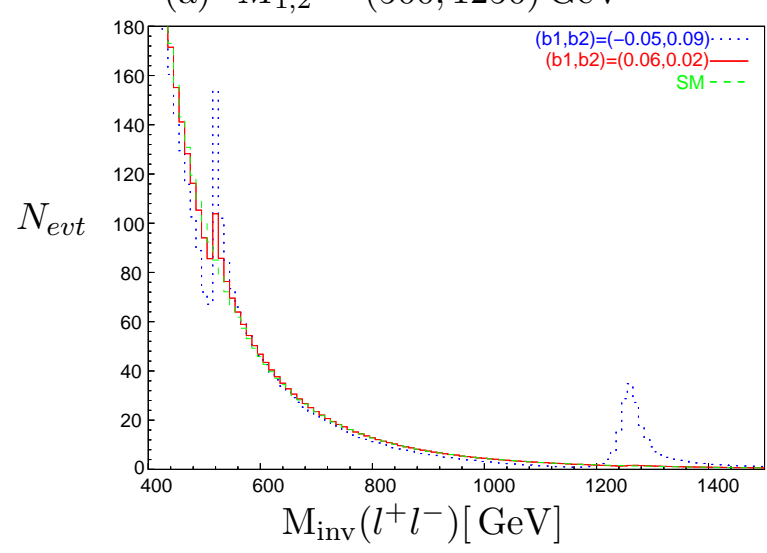

(c) $M_{1,2}=(1000,1250) \mathrm{GeV}$

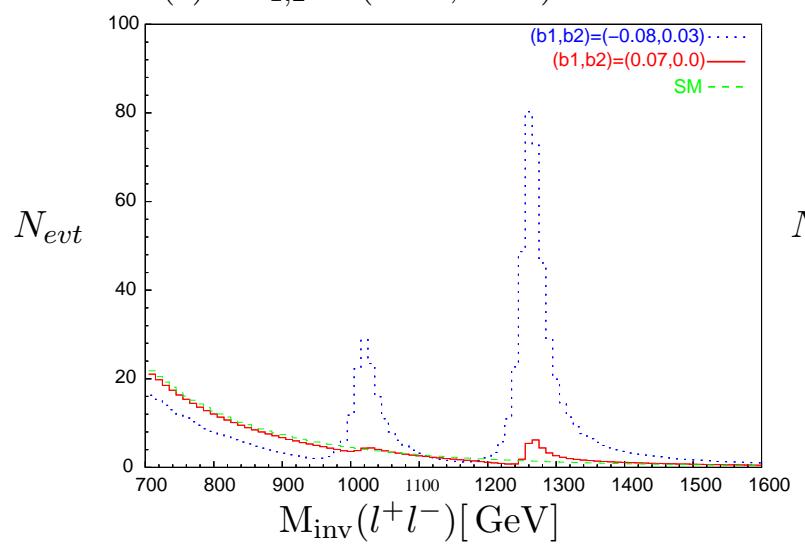

(b) $M_{1,2}=(1732,3000) \mathrm{GeV}$

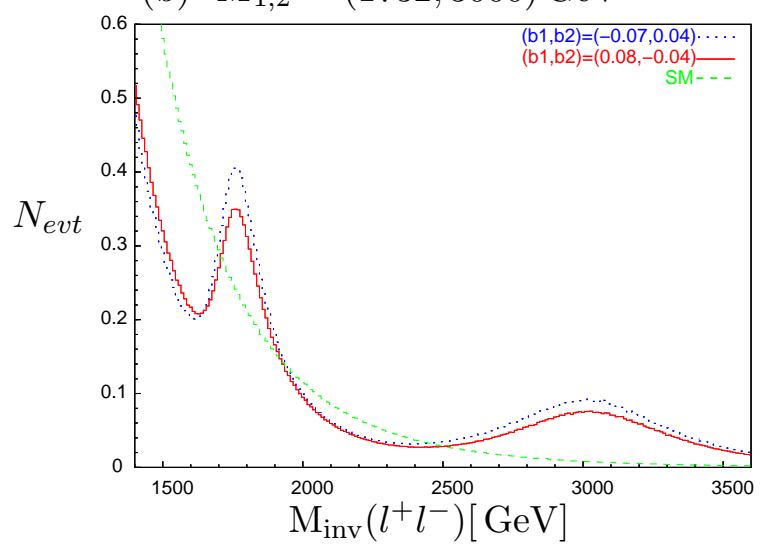

(d) $M_{1,2}=(1000,1250) \mathrm{GeV}$

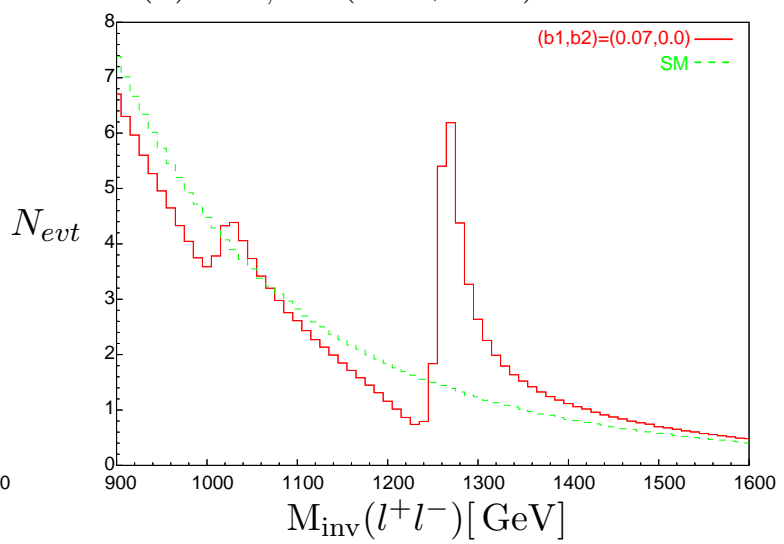

FIG. 5: Total number of events in a $10 \mathrm{GeV}$-bin versus the dilepton invariant mass, $M_{\mathrm{inv}}\left(l^{+} l^{-}\right)$, for the process pp $\rightarrow l^{+} l^{-}$at the integrated luminosity $L=10 \mathrm{fb}^{-1}$ for the six scenarios of Table I. We sum over $e, \mu$. Standard cuts and legends as in the text.

$(0.4,1 / \sqrt{3}, 0.8)$, and various $b_{1,2}$-sets. The corresponding neutral fermion-boson couplings are summarized in Table $\mathrm{V}$ of Appendix $\mathrm{B}$.

We start from the spectrum of the neutral extra gauge bosons as it could appear in the Drell-Yan channel at the LHC. In Fig. 5, we plot the total number of events as a function of the dilepton invariant mass, $M_{\mathrm{inv}}\left(l^{+} l^{-}\right)$, for the six aforementioned scenarios. We have checked that all these cases are outside the exclusion limit from direct searches at the Tevatron with an integrated luminosity $\mathrm{L}=4 \mathrm{fb}^{-1}$ [58]. From top to bottom, the three curves in each plot represent the first $b_{1,2}$-setup, the latter, and the SM prediction at fixed $M_{1,2}$ masses. We sum over $e, \mu$ and apply standard acceptance cuts. 
These examples show four peculiarities of the model. First of all, the masses of the two neutral resonances $Z_{1,2}$ are not equally spaced. They can be either very distant for low $z$ values (see Figs. $5 \mathrm{~b}$ and $5 \mathrm{~b}$ ), or they can tend to be almost degenerate for high $z$-values (see Fig. 5k). For some values of the $b_{1,2}$-parameter (see for example the case 6 ), one of the two resonances could also disappear leaving a single-resonant (or even not-resonant) spectrum like in Fig. 5 $5 \mathrm{~d}$. A second feature is related to the interference between signal and SM background. All four plots exhibit indeed a sizeable depletion of the total number of events, compared to the SM prediction, in the off-peak region. This characteristic is particularly evident by increasing the new gauge boson masses (see Figs. $5 \mathrm{~b}$ and $5 \mathrm{5}$ ), and improves the detection rate. A further distinctive behaviour is represented by the width magnitude. It indeed increases with the fifth power of the extra gauge boson mass. One can thus pass from configurations with very narrow resonances, see Fig. 5 b, to scenarios characterized by broad peaks, or even shoulders as in Fig. 5b. The last feature concerns the relative size of the $Z_{1,2}$ resonances. Tab. $\mathrm{V}$ in Appendix $\mathrm{B}$ shows that in most part of the parameter space the $Z_{1}$-fermion couplings are smaller than the $Z_{2}$-fermion ones. As a consequence, the height of the $Z_{1}$-resonance is less pronounced than the $Z_{2}$-peak. This feature can be washed out by the PDF effect, but it is clearly visible in Fig. 5 k.

In order to have an idea of the detection rate expected at the LHC for the Drell-Yan production of the extra $Z_{1,2}$ gauge bosons, in Tab. II we have listed signal and total event number in the two distinct on-peak regions $\left|M_{\text {inv }}\left(l^{+} l^{-}\right)-M_{1,2}\right|<\Gamma_{1,2}$ for the six considered scenarios. From Tab. II it is clear that while the second $b_{1,2}$-setup, at each fixed $M_{1,2}$ mass, would need high luminosity to be detected, the first $b_{1,2}$-setup could already be visible at the LHC start-up with a luminosity $\mathrm{L} \simeq 1 \mathrm{fb}^{-1}$.

In presence of two resonances, the four site Higgsless model could not be easily misidentified. However, in the case depicted in Fig. 5 5 d where only one resonance survives, the model-degeneracy problem fully arises. Many models predict in fact an extra neutral vector boson, one for all the sequential SM (SSM). Disentangling the various theories, and tracing back the lagrangian parameters is not an easy task. To this end, a useful observable, longly studied in the literature, is represented by the forward-backward charge asymmetry $A_{F B}$. The sensitivity of $A_{F B}$ measurements to new physics like additional $Z^{\prime}$-bosons has been discussed by several authors [59, 60]. For leptonic Drell-Yan processes, $A_{F B}$ is defined from 


\begin{tabular}{|c|c|c|c|c|c|c|c|c|}
\hline & $M_{1,2}(\mathrm{GeV})$ & $b_{1,2}$ & $N_{\text {evt }}^{\text {sig }}\left(Z_{1}\right)$ & $N_{\text {evt }}^{\text {tot }}\left(Z_{1}\right)$ & $\sigma\left(Z_{1}\right)$ & $N_{\text {evt }}^{\text {sig }}\left(Z_{2}\right)$ & $N_{\text {evt }}^{\text {tot }}\left(Z_{2}\right)$ & $\sigma\left(\mathrm{Z}_{2}\right)$ \\
\hline \hline 1 & 500,1250 & $-0.05,0.09$ & 47 & 154 & 3.8 & 134 & 143 & 11.2 \\
\hline 2 & 500,1250 & $0.06,0.02$ & 11 & 123 & 1.0 & 0 & 9 & 0.0 \\
\hline 3 & 1732,3000 & $-0.07,0.04$ & 7 & 10 & 2.2 & 7 & 8 & 2.5 \\
\hline 4 & 1732,3000 & $0.08,-0.04$ & 5 & 9 & 1.7 & 6 & 6 & 2.4 \\
\hline 5 & 1000,1250 & $-0.08,0.03$ & 108 & 119 & 9.9 & 291 & 302 & 16.7 \\
\hline 6 & 1000,1250 & $0.07,0.0$ & 3 & 28 & 0.0 & 15 & 22 & 3.2 \\
\hline
\end{tabular}

TABLE II: The first three columns represent the scenario. The next three columns give signal and total (including the SM background) event number for the $Z_{1}$ production, and the statistical significance $\sigma=N_{\mathrm{evt}}^{\text {sig }} / \sqrt{N_{\mathrm{evt}}^{\mathrm{tot}}}$ for an integrated luminosity $\mathrm{L}=10 \mathrm{fb}^{-1}$. The last three columns show the same results for the $Z_{2}$ production.

the angular distribution with respect to the quark direction

$$
\frac{d \sigma}{d \cos \theta_{l}^{*}} \propto \frac{3}{8}\left(1+\cos ^{2} \theta_{l}^{*}\right)+A_{F B} \cos \theta_{l}^{*}
$$

where $\theta_{l}^{*}$ is the lepton $(e, \mu)$ angle in the dilepton center-of-mass frame $(\mathrm{CM})$, which can be derived from the measured four-momenta of the dilepton system in the laboratory frame. As in pp collisions the original quark direction is not known, one has to extract it from the kinematics of the dilepton system. In this analysis, we follow the criteria of Ref. [60] and simulate the quark direction from the boost of the dilepton system with respect to the beam axis. As a measure of the boost, we define the dilepton rapidity $y=1 / 2 \times \ln \left[\left(E+p_{z}\right) /(E-\right.$ $\left.p_{z}\right)$ ], and identify the quark direction through the sign of $y$. We further impose the rapidity to be bigger than one, $|y| \geq 1$. This cut ensures that the fraction of high mass dilepton events with a correctly assigned quark direction is around $80 \%$.

We study both on-resonance and off-resonance asymmetries for the single-resonant scenario depicted in Fig. $5 \mathrm{~d}$. In the first case, we consider the on-peak region $\left|M_{\mathrm{inv}}\left(l^{+} l^{-}\right)-M_{2}\right|<$ $3 \Gamma_{2}$, and plot the angular distribution of Eq. (47), including signal and background. In order to illustrate how $A_{F B}$ could help in solving the model-degeneracy problem, in Fig. 6a we compare the four site Higgsless model with the sequential SM, which both predict an extra neutral vector boson $\left(Z_{2}\right)$. The SM result is given as a reference. As one can see, the two models present quite a different shape for the differential cross section in $\cos \theta_{l}^{*}$. The 

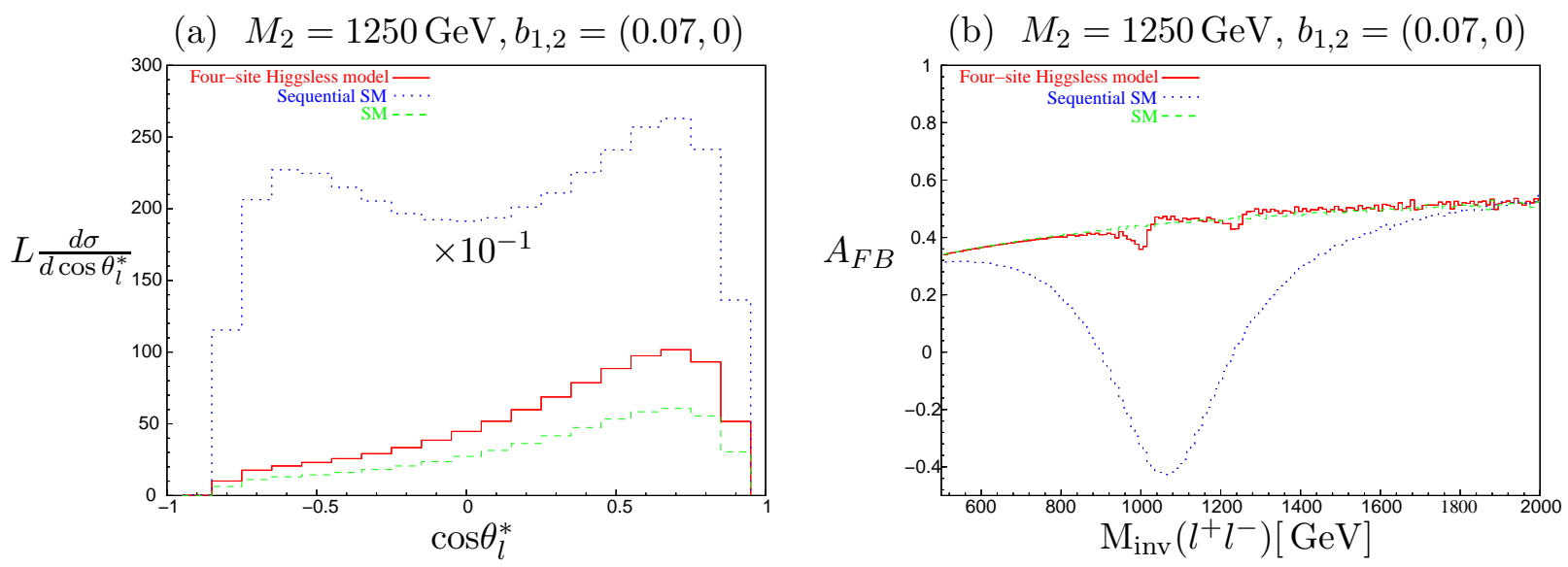

FIG. 6: (a) Angular distribution in the lepton angle with respect to the quark direction in the dilepton rest frame, for the process $\mathrm{pp} \rightarrow l^{+} l^{-}$at the integrated luminosity $\mathrm{L}=100 \mathrm{fb}^{-1}$. From top to bottom, the three curves describe a sequential-SM $Z^{\prime}$-boson with mass $M_{2}=1250 \mathrm{GeV}$, a four site Higgsless $Z_{2}$-boson with same mass $M_{2}=1250 \mathrm{GeV}$ and $b_{1,2}=(0.07,0)$, and the SM prediction. (b) Off-resonance forward-backward asymmetry versus the dilepton invariant mass, $M_{\text {inv }}\left(l^{+} l^{-}\right)$, for the process pp $\rightarrow l^{+} l^{-}$at the integrated luminosity $\mathrm{L}=100 \mathrm{fb}^{-1}$. From top to bottom, the three curves represent the SM prediction, a four site Higgsless $Z_{2}$-boson with mass $M_{2}=1250 \mathrm{GeV}$ and $b_{1,2}=(0.07,0)$, and a sequential-SM $Z^{\prime}$-boson with mass $M=1250 \mathrm{GeV}$. In both figures, we sum over $e, \mu$. Standard cuts and legends as in the text.

forward-backward asymmetry is much more pronounced in the four site Higgsless model, owing to the sensible difference between left and right-handed fermion-boson couplings, as reported in Tab. $\mathrm{V}$ of Appendix B. The coefficient, $A_{F B}$, in Eq. (47) is indeed proportional to the difference between the two couplings squared, $A_{F B} \propto\left[\left(a_{2 L}^{f}\right)^{2}-\left(a_{2 R}^{f}\right)^{2}\right]$.

As a further tool to separate the two models, in Fig. 6b we plot the asymmetry for continuum dilepton events at high CM energy scales. Here, we integrate over the lepton angle in the forward and backward region, separately, and plot the difference between the resulting forward and backward differential cross sections in the dilepton invariant mass, normalized to their sum

$$
A_{F B}=\left[\frac{d \sigma^{F}}{d M_{\mathrm{inv}}}-\frac{d \sigma^{B}}{d M_{\mathrm{inv}}}\right] /\left[\frac{d \sigma^{F}}{d M_{\mathrm{inv}}}+\frac{d \sigma^{B}}{d M_{\mathrm{inv}}}\right] .
$$



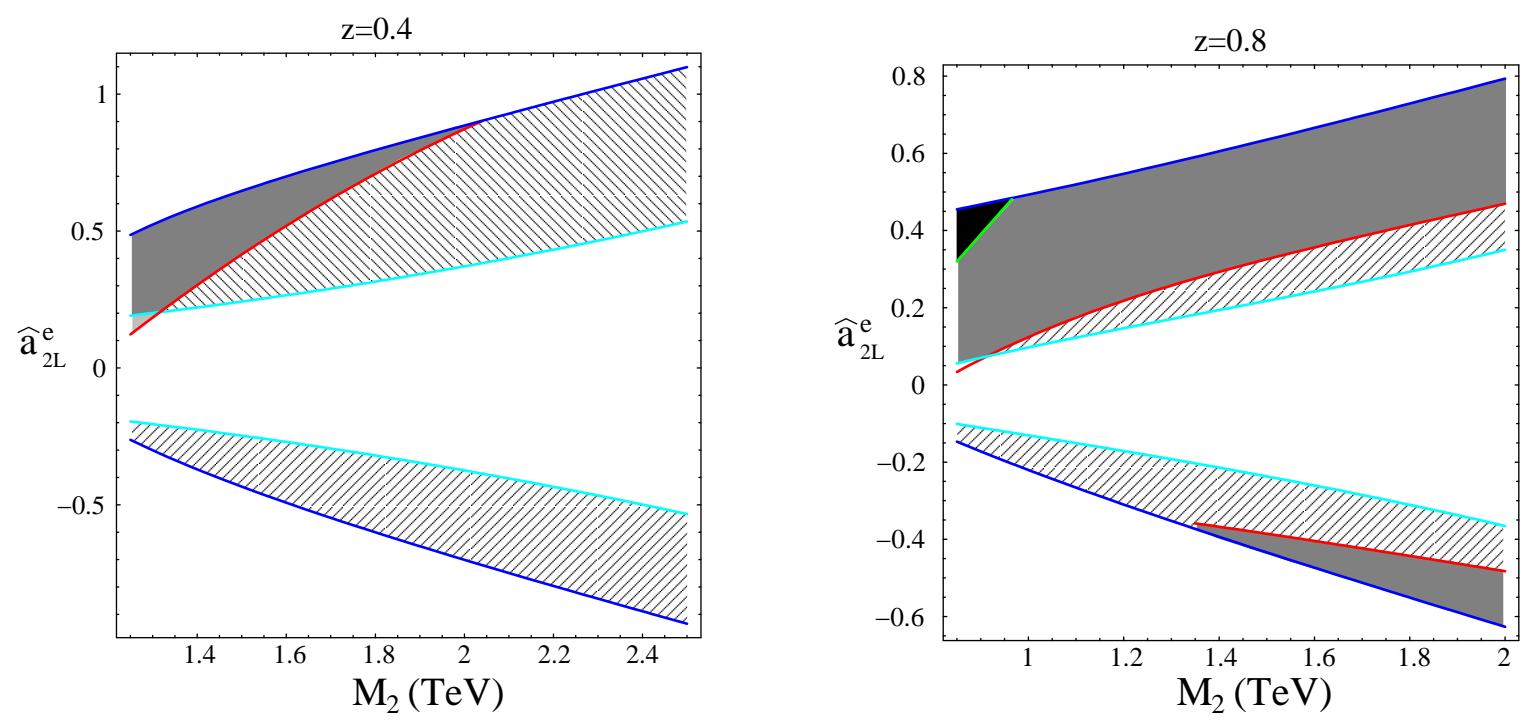

FIG. 7: $5 \sigma$-discovery plot at $\mathrm{L}=100 \mathrm{fb}^{-1}$ in the plane $\left(\hat{a}_{2 L}^{e}, M_{2}\right)$ (in the coupling the electric charge - $e$ is factorized). The upper and lower parts are excluded by EWPT, the black triangle in the right panel is the region excluded by the direct search at the Tevatron. Inside the dark-grey regions both $Z_{1,2}$ are visible; inside the grey (dashed) ones only $Z_{1}\left(Z_{2}\right)$ can be detected. Inside the central uncolored region no resonance is visible in the Drell-Yan channel. Left (right) panel: $z=0.4$ $(z=0.8)$.

The SM prediction is shown as a reference. The measurement of the dips, pointing at different CM-energy values according to the considered model, represents an additional powerful tool to understand the nature of the neutral resonance. In this particular case, the off-resonance $A_{F B}$ could also reveal the double-resonant structure of the four site Higgsless model signal, not appreciable in the dilepton invariant mass distribution (compare with Fig. 5d). The first dip, related to the $Z_{1}$-boson mass, gives indeed a $3.8 \sigma$ effect if compared to the SM prediction, for an high luminosity $\mathrm{L}=100 \mathrm{fb}^{-1}$.

After analyzing the spectrum of the extra $Z_{1,2}$-bosons, and how their nature could be investigated through asymmetry measurements, let us summarize the possibility to detect these new particles at the LHC as a function to the integrated luminosity. In Fig. 7 we plot the $5 \sigma$-discovery contours at $\mathrm{L}=100 \mathrm{fb}^{-1}$ in the plane $\left(\hat{a}_{2 L}^{e}, M_{2}\right)$, where $\hat{a}_{2 L}^{e}$ is the left-handed coupling between the $Z_{2}$-boson and the SM electron (in -e units) and $M_{2}$ is related to the $Z_{2}$-mass by Eq. (A16). We have considered $M_{2} \leq 2(2.5) \mathrm{TeV}$ for $z=0.8(0.4)$ in order to agree with the strongest partial wave unitarity bound shown in Fig. 4 and two different 

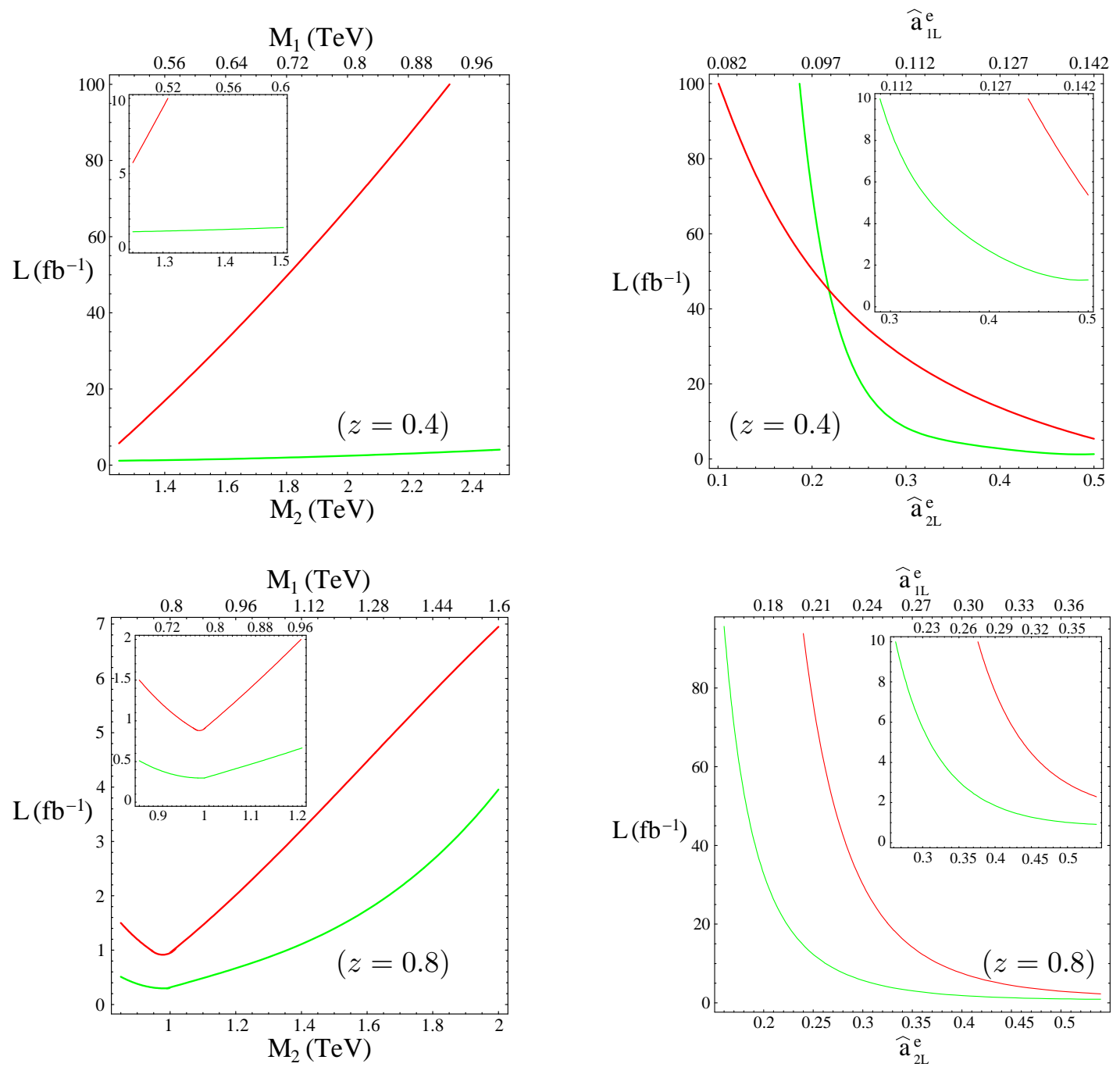

FIG. 8: Left panels: Luminosity vs $Z_{2}$ mass needed for a $5 \sigma$-discovery for $z=0.4$ (up) and $z=0.8$ (down). We assume the maximum value for the fermion-boson couplings allowed by EWPT and Tevatron, as shown by the upper curves in Fig. 17. The discovery curves are for $Z_{1}$ (red, darker) and $Z_{2}$ (green, lighter) gauge bosons. Right panels: Luminosity vs electron-boson left-handed coupling (the electric charge $-e$ is factorized) needed for a $5 \sigma$-discovery for $z=0.4$ (up) and $z=0.8$ (down) with $M_{2}=1250 \mathrm{GeV}$ and $M_{1}=z M_{2}$. The discovery curves are for $Z_{1}$ (red, darker) and $Z_{2}$ (green, lighter) gauge bosons. In all the figures, we sum over $e, \mu$ and apply standard cuts. The inset plots show the zoomed-in low-luminosity region.

values of the parameter $z$. The coupling $a_{1 L}^{e}$ is fixed by using the expression of $\epsilon_{3}$ given in Eq. (33) after including radiative corrections, and comparing it to the experimental value, $\epsilon_{3}^{e x p}$. Looking at the upper part of the plots and going from top to bottom, the first curve represents the $\hat{a}_{2 L}^{e}$ maximum values allowed by EWPT as a function of $M_{2}$. The small black 
triangle in the right panel gives the direct limits from Tevatron for a luminosity $L=4 \mathrm{fb}^{-1}$ [58]. This region is not visible in the left panel because of the different mass range. As to the detection, the dark-grey region between the first two curves represents the parameter space where both $Z_{1,2}$ resonances are simultaneously visible in the Drell-Yan channel, while the dashed region shows the range where only the $Z_{2}$-boson could be detected. The small grey region for low masses shows the range where only the $Z_{1}$-boson could be detected. The lower part of the plot is specular. In the central uncolored region one should measure other channels like di-boson production and $W W$ scattering. Notice that for low $z$ values, the $Z_{1^{-}}$ boson couplings to fermions are much more bounded by EWPT (Eq. (33)), as a consequence the $Z_{1}$ visibility region by DY production is smaller with respect to larger $z$ values.

The main information one gets from Fig. 7 is that the four site Higgsless model can be explored at the LHC in the favoured Drell-Yan channel, over a large portion of the parameter space, without invoking much more complex multi-particle processes like di-boson production or vector boson fusion as in the usual Higgsless literature [34, 35]. Moreover, the new $Z_{1,2}$ gauge bosons could be discovered in the early stage of the LHC data taking at very low luminosity.

Fig. 8 (left panels) shows in fact that the minimum integrated luminosity required to observe the low edge of the spectrum is around $\mathrm{L} \simeq 1-2 \mathrm{fb}^{-1}$. Notice that the raise of luminosity for low masses for $z=0.8$ is due to the Tevatron exclusion region shown in Fig. 7 , In Fig. 8 (right panels), we plot in addition the luminosity needed for a $5 \sigma$ discovery of a $Z_{2}$-boson with mass $M_{2}=1.25 \mathrm{TeV}$ and a $Z_{1}$-boson with mass $M_{1}=z M_{2}$ as a function of the left-handed electron-boson coupling.

\section{2. $W_{1}^{ \pm}$and $W_{2}^{ \pm}$production at the $L H C$}

In this section, we present some cross sections and distributions for the leptonic process $\mathrm{pp} \rightarrow l \nu_{l}$ with $l=\mathrm{e}, \mu$ and $l \nu_{l}=l^{-} \bar{\nu}_{l}, l^{+} \nu_{l}$. These final states allow to analyze the production of the four charged extra gauge bosons, $W_{1,2}^{ \pm}$, predicted by the four site Higgsless model.

As we said previously, we are interested in the high energy region where the new strongly interacting vector bosons are expected to be produced. We thus impose an additional cut on the transverse momentum of the lepton pair, $P_{T}\left(l \nu_{l}\right) \geq 150 \mathrm{GeV}$, which selects large CM-energies $(\sqrt{s} \geq 300 \mathrm{GeV})$. In order to illustrate spectrum and behaviour of the new 

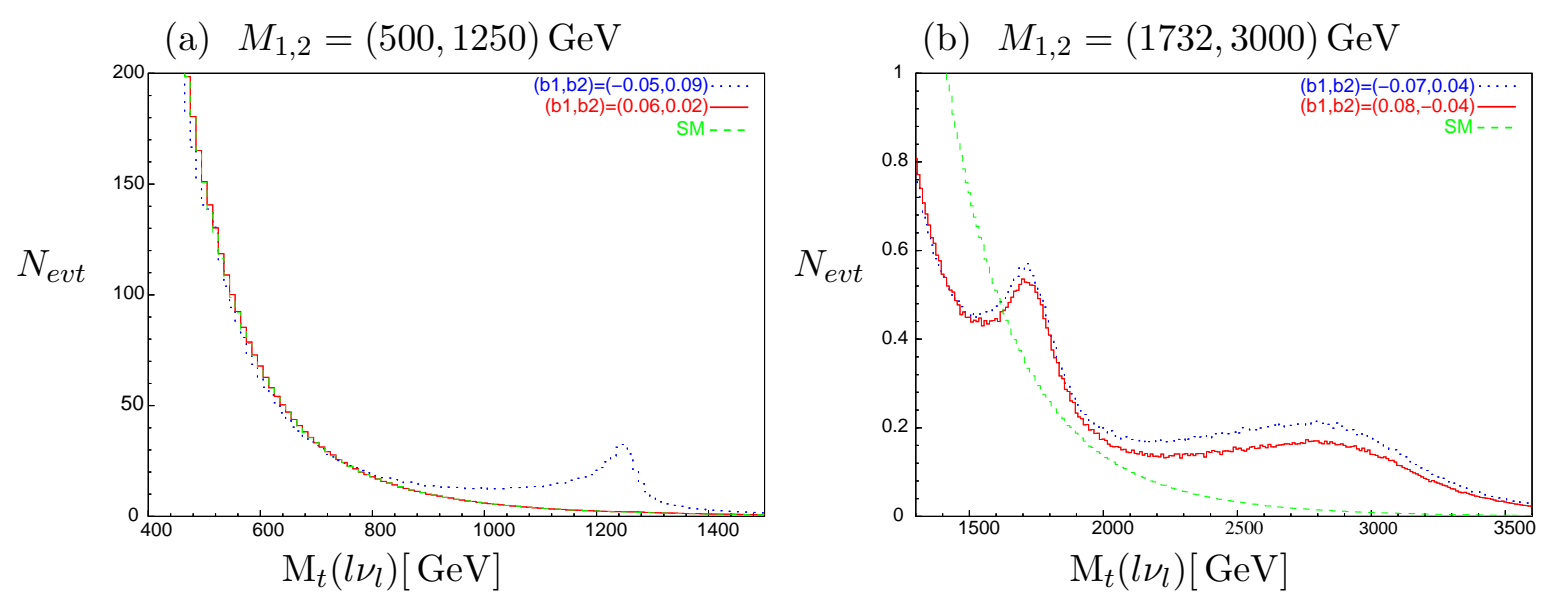

(c) $M_{1,2}=(1000,1250) \mathrm{GeV}$

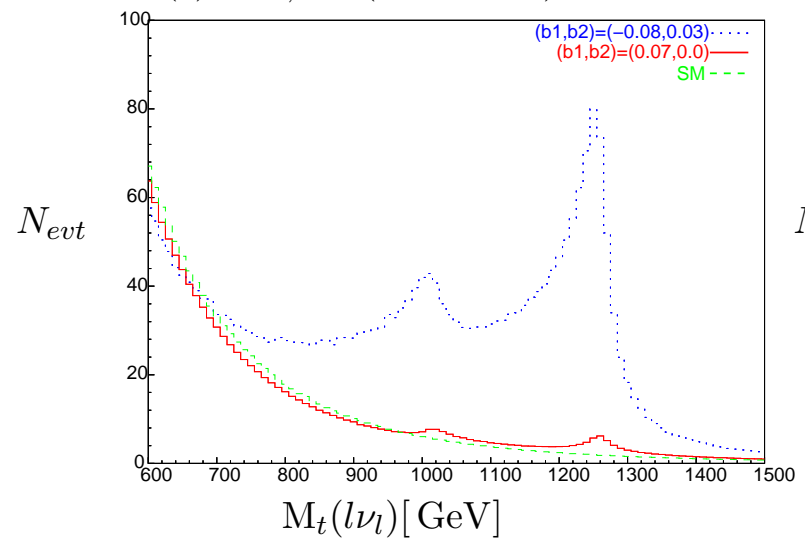

(d) $M_{1,2}=(1000,1250) \mathrm{GeV}$

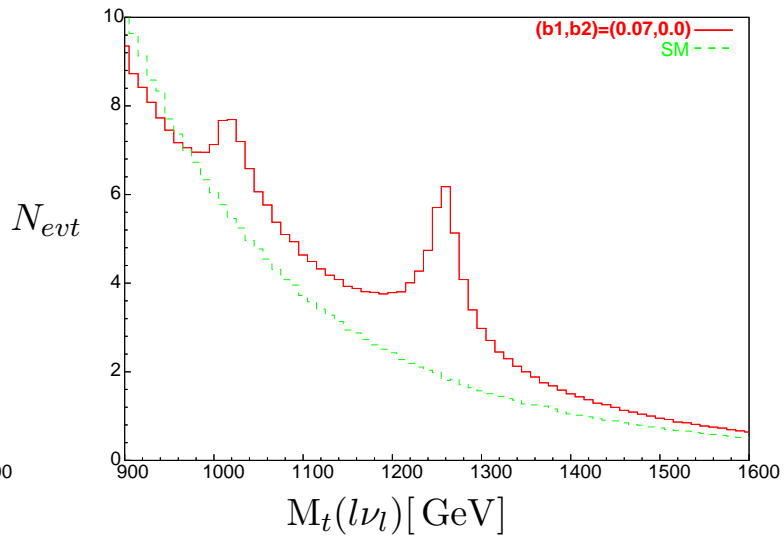

FIG. 9: Total number of events in a $10 \mathrm{GeV}$-bin versus the lepton transverse mass, $M_{t}\left(l \nu_{l}\right)$, for the process pp $\rightarrow l \nu_{l}$ at the integrated luminosity $L=10 \mathrm{fb}^{-1}$ for the six scenarios of Table【. We sum over $e, \mu$ and charge conjugate channels. Standard cuts and legends as in the text.

charged particles at the LHC, we have chosen to analyze the distribution in the transverse mass of the lepton pair, $M_{t}\left(l \nu_{l}\right)$, for the six scenarios of Tab. I, that is for three values of the $z$-parameter, $z=(0.4,1 / \sqrt{3}, 0.8)$, and various $b_{1,2}$-sets. The corresponding charged fermion-boson couplings are summarized in Tab. [V] of Appendix [B.

In analogy with the neutral case, in Fig. 9 we plot the total number of events as a function of the dilepton transverse mass, $M_{t}\left(l \nu_{l}\right)$, for the six aforementioned scenarios. From top to bottom, the three curves in each plot represent the first $b_{1,2}$-setup, the latter, and the SM prediction at fixed $M_{1,2}$ mass parameter. We sum over $e, \mu$ and charge conjugate processes. We moreover apply standard acceptance cuts. 


\begin{tabular}{|c|c|c|c|c|c|c|c|c|c|}
\hline & $M_{1,2}(\mathrm{GeV})$ & $b_{1,2}$ & $M_{t}^{\text {cut }}(\mathrm{GeV})$ & $N_{\text {evt }}^{\text {sig }}\left(W_{1}\right)$ & $N_{\text {evt }}^{\text {tot }}\left(W_{1}\right)$ & $\sigma\left(W_{1}\right)$ & $N_{\text {evt }}^{\text {sig }}\left(W_{2}\right)$ & $N_{\text {evt }}^{\text {tot }}\left(W_{2}\right)$ & $\sigma\left(W_{2}\right)$ \\
\hline \hline 1 & 500,1250 & $-0.05,0.09$ & 400 & 36 & 2435 & 0.7 & 776 & 2214 & 16.5 \\
\hline 2 & 500,1250 & $0.06,0.02$ & 400 & 0 & 2609 & 0 & 1 & 1807 & 0 \\
\hline 3 & 1732,3000 & $-0.07,0.04$ & 1500 & 10 & 18 & 2.4 & 24 & 26 & 4.7 \\
\hline 4 & 1732,3000 & $0.08,-0.04$ & 1500 & 9 & 14 & 2.4 & 22 & 24 & 4.5 \\
\hline 5 & 1000,1250 & $-0.08,0.03$ & 700 & 808 & 1230 & 23.0 & 1112 & 1189 & 32.3 \\
\hline 6 & 1000,1250 & $0.07,0.0$ & 700 & 12 & 443 & 0.6 & 17 & 88 & 1.8 \\
\hline
\end{tabular}

TABLE III: The first three columns represent the scenario. The fourth one shows the cut on the dilepton transverse mass $M_{t}\left(l \nu_{l}\right)$. The next three columns give signal and total (including the SM background) event number for the $W_{1}^{ \pm}$production, and the statistical significance $\sigma=N_{\text {evt }}^{\text {sig }} / \sqrt{N_{\text {evt }}^{\text {tot }}}$ for an integrated luminosity $\mathrm{L}=10 \mathrm{fb}^{-1}$. The last three columns give the corresponding results for $W_{2}^{ \pm}$production.

These examples show again some peculiar predictions of the model. First of all, the spectra of neutral and charged gauge sectors are almost degenerate $\left(M_{i, c} \simeq M_{i, n}\right.$ with $i=$ 1,2). The same peaking structure is thus expected in both neutral and charged Drell-Yan channels. This is clearly visible in Figs. 9b,c where the peaks in the lepton transverse mass are centered on the same values as those ones in the dilepton invariant mass reported in Figs. 5b,c. In these cases, we have a cross-checkable double signal. The situation can however be different, depending on masses and couplings. For example, if one considers the first scenario of Tab. I, one realizes that while in the neutral channel the two new resonances are both visible (at least at parton level), in the charged channel only the heavier one survives. The first $W_{1}^{ \pm}$-boson peak is indeed washed out by the very low couplings between charged extra gauge bosons and SM fermions (see Tab. IV in Appendix [B). In this case, in order to have the full information, exploring the two channels is mandatory. The last plot in Fig. 9d is again a mirror of Fig. 5d, i.e. in this scenario only the heavier resonances $W_{2}^{ \pm}$and $Z_{2}$ could be detected in their respective channels (with $L \sim 100 \mathrm{fb}^{-1}$ ).

In order to estimate the detection rate expected at the LHC for the Drell-Yan production of the extra $W_{1,2}^{ \pm}$gauge bosons, in Tab. III we have listed signal and total event number for the six scenarios given in Tab. I] We have evaluated the number of $W_{1,2}^{ \pm}$events in the 

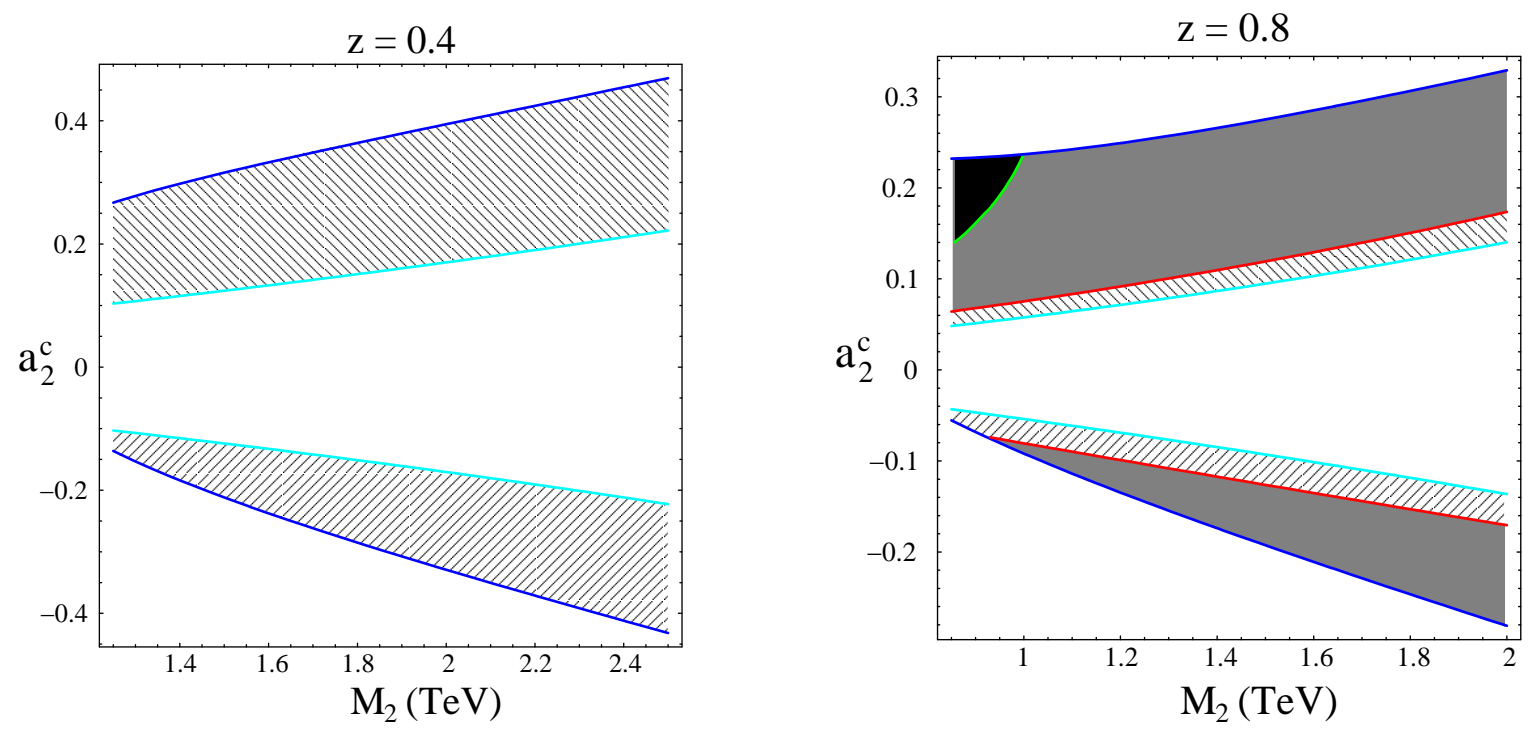

FIG. 10: $5 \sigma$-discovery plot at $\mathrm{L}=100 \mathrm{fb}^{-1}$ in the plane $\left(a_{2}^{c}, M_{2}\right)$. The upper and lower parts are excluded by EWPT, the black triangle is the region excluded by the direct search at the Tevatron. Inside the grey regions both $W_{1,2}$ are visible; inside the dashed ones only $W_{2}$ can be detected. Inside the central uncolored region no resonance is visible in the Drell-Yan channel. Left (right) panel: $z=0.4(z=0.8)$

kinematical region defined by the cut: $M_{t}\left(l \nu_{l}\right) \geq M_{t}^{c u t}$. The value of $M_{t}^{\text {cut }}$ is chosen as the value where the total number of events is equal to the SM background. The number of events is obtained by integrating in transverse mass between $M_{t}^{c u t}$ and $M_{1}+\Gamma_{1}$ for the first resonance and between $M_{1}+\Gamma_{1}$ and $M_{2}+\Gamma_{2}$ for the second one. ¿From Tab. III, one can see that the charged Drell-Yan channel has generally a higher sensitivity compared to the neutral one, for a given scenario. In some cases the statistical significance is about a factor two bigger than the previous neutral case, however the charged Drell-Yan observables are not that clean. In order to have a well defined information on the four site Higgsless model predictions, neutral and charged Drell-Yan channels are thus complementary. And, more important, there are regions in the parameter space where they could be both investigated for the search of all six extra gauge bosons, $W_{1,2}^{ \pm}$and $Z_{1,2}$, at the LHC start-up with a luminosity of the order of $\mathrm{L} \simeq 1-2 \mathrm{fb}^{-1}$ for $M_{1,2} \leq 1 \mathrm{TeV}$.

Let us finally comment on the dependence of the discovery potential in the charged channel in the parameter space of the model. In Fig. 10 we plot the $5 \sigma$-discovery contours at $\mathrm{L}=100 \mathrm{fb}^{-1}$ for $z=0.4$ (left) and $z=0.8$ (right) in the plane $\left(a_{2}^{c}, M_{2}\right)$ where $a_{2}^{c}$ is the 
$W_{2}$ charged coupling and $M_{2}$ is related to the $W_{2}$ mass by Eq. (A5). The upper and lower parts of the plot are excluded by EWPT, the black triangle is the region excluded by the direct search at the Tevatron with $4 \mathrm{fb}^{-1}$. Inside the dark-grey regions both $W_{1,2}$ are visible; inside the dashed ones only $W_{2}$ can be detected. Inside the central uncolored region no resonance is visible in the Drell-Yan channel. Notice that, while for $z=0.8$ the region in which one looses the first resonance is small, for smaller $z$-values this region increases and for example, for $z=0.4$, the four site model could be misidentified with schemes with only one triplet of new resonances. For this portion of parameter space the di-boson and/or the fusion channels are mandatory for disentangling among different models.

\section{CONCLUSIONS}

In this work we have studied the phenomenological consequences of the four site Higgsless model, a deconstructed theory which can derive from the discretization of the fifth dimension on a lattice, and is based on the $S U(2)_{L} \times S U(2)_{1} \times S U(2)_{2} \times U(1)_{Y}$ gauge symmetry. The model represents an extension of the minimal three site version (or BESS model), longly investigated in the literature, which includes three heavy vector bosons, $W_{1}^{ \pm}$and $Z_{1}$. The four site model extends the gauge sector to four charged and two neutral extra gauge bosons, $W_{1,2}^{ \pm}$and $Z_{1,2}$. We have analysed their properties and the prospects for their direct search at the LHC.

One of the strong motivations for Higgsless models is their ability to delay the unitarity violation of VBS amplitudes to energy scales higher than those predicted by the SM without a light Higgs, just via the exchange of the above mentioned extra gauge bosons. The drawback is the strong tension between the unitarity requirement and the bounds coming from the electroweak precision tests. In the minimal three site model, the request to reconcile unitarity and EWPT brings to a fermiophobic scenario, where the extra gauge bosons can barely interact with ordinary matter. In this case, the only production processes available to search for the new particles are those dominated by boson-boson interactions. Hence, one has to rely on difficult multi-particle processes which require high luminosity to be revealed, that is vector boson fusion and triple gauge boson production. The recent Higgsless literature has been focused on this side.

The novelty of the four site Higgsless model consists in reconciling unitarity and EWPT 
bounds without imposing the extra vector bosons to be fermiophobic, owing to the inclusion of direct fermion-boson couplings in addition to those ones coming from usual mixing terms. We have analysed in detail the constraints on masses and couplings of the extra gauge bosons coming from unitarity requirement and EW precision data consistency. We have found that, asking for all VBS amplitudes with both SM and extra gauge bosons as external states to be unitarized, the perturbative regime can be extended up to energy scales of the order of $\sqrt{s} \simeq 3 \mathrm{TeV}$, thus higher than those predicted by the SM with no light Higgs and by the three site minimal version. Hence, the spectrum of the new gauge bosons must lie within a few $\mathrm{TeV}$, a region which represents the main effective energy range available at the LHC. We have moreover investigated the impact of the EW precision measurements expressed in terms of the $\epsilon_{i}$ parameters on the couplings of the extra gauge bosons to ordinary matter. While $\epsilon_{3}$ gives the strongest bound, imposing a strict relation between the couplings of the two vector boson triplets, $\epsilon_{1}$ weakly limits their size owing to the $\mathrm{SU}(2)$ custodial symmetry ( $\epsilon_{2}$ is uneffective). The fermion-boson couplings can be thus of the same order of the SM ones. As a consequence, the Drell-Yan process becomes an open channel for the direct search of the extra gauge bosons at the LHC.

We have analysed in detail the potential detection rate of the new particles, evaluating cross sections and distributions for charged and neutral Drell-Yan processes at the LHC. In order to show a full view, we have considered different possible scenarios predicted by the four site Higgsless model. There, the resonances are not equally spaced; so we have studied the spectrum, going from very spaced cases to almost degenerate ones.

The outcome is that all six extra gauge bosons could be detected at the LHC over a large portion of the parameter space (i.e. couplings and masses) for a luminosity at regime. The low-edge mass spectrum, below $1 \mathrm{TeV}$, could be already discovered during the early stage of the LHC data taking, with a start-up luminosity of the order of $L \simeq 1 \mathrm{fb}^{-1}$.

These results do not include the detector simulation. At present, this work is in progress, but we do not expect a drastic change in our conclusions owing to the very clean signals.

\section{APPENDIX A: GAUGE BOSON SPECTRUM}

The charged gauge boson mass Lagrangian is given by:

$$
\mathcal{L}_{\text {mass }}^{\mathcal{C}}=\tilde{\mathcal{C}}^{-} \mathcal{M}_{c}^{2} \tilde{\mathcal{C}}^{+}
$$


with $\tilde{\mathcal{C}^{-}}=\left(\tilde{W}^{-}, \tilde{A}_{1}^{-}, \tilde{A}_{2}^{-}\right)$

$$
\mathcal{M}_{c}^{2}=\left(\begin{array}{ccc}
\tilde{g}^{2} f_{1}^{2} & -\tilde{g} g_{1} f_{1}^{2} & 0 \\
-\tilde{g} g_{1} f_{1}^{2} & g_{1}^{2}\left(f_{1}^{2}+f_{2}^{2}\right) & -g_{1}^{2} f_{2}^{2} \\
0 & -g_{1}^{2} f_{2}^{2} & g_{1}^{2}\left(f_{1}^{2}+f_{2}^{2}\right)
\end{array}\right)
$$

The eigenvalues for large $g_{1}$, neglecting $\mathcal{O}\left(\tilde{g}^{4} / g_{1}^{4}\right)$, have the following expressions:

$$
\begin{gathered}
M_{W}^{2} \approx \tilde{M}_{W}^{2}\left(1-\frac{\tilde{g}^{2}}{g_{1}^{2}} z_{W}\right) \\
M_{1, c}^{2} \approx M_{1}^{2}\left(1+\frac{\tilde{g}^{2}}{2 g_{1}^{2}}\right) \\
M_{2, c}^{2} \approx M_{2}^{2}\left(1+\frac{\tilde{g}^{2}}{2 g_{1}^{2}} z^{4}\right)
\end{gathered}
$$

with the identifications

$$
\begin{aligned}
& \tilde{M}_{W}^{2}=\tilde{g}^{2} \frac{f_{1}^{2} f_{2}^{2}}{f_{1}^{2}+2 f_{2}^{2}} \quad z_{W}=\frac{f_{1}^{4}+2 f_{1}^{2} f_{2}^{2}+2 f_{2}^{4}}{\left(f_{1}^{2}+2 f_{2}^{2}\right)^{2}}=\frac{1}{2}\left(1+z^{4}\right) \\
& M_{1}^{2}=f_{1}^{2} g_{1}^{2} \quad M_{2}^{2}=g_{1}^{2}\left(f_{1}^{2}+2 f_{2}^{2}\right) \quad z=\frac{M_{1}}{M_{2}}=\frac{f_{1}}{\sqrt{f_{1}^{2}+2 f_{2}^{2}}}
\end{aligned}
$$

We give also, at the same order, the transformations which express the fields $\tilde{W}^{ \pm}, \tilde{A}_{1}^{ \pm}$and $\tilde{A}_{2}{ }^{ \pm}$in terms of the mass eigenvalues

$$
\begin{aligned}
\tilde{W}^{ \pm}= & \left(1-\frac{\tilde{g}^{2}}{g_{1}^{2}} \frac{z_{W}}{2}\right) W^{ \pm} \\
& -\frac{\tilde{g}}{\sqrt{2} g_{1}}\left(1+\frac{\tilde{g}^{2}}{4 g_{1}^{2}} \frac{1-3 z^{2}}{1-z^{2}}\right) W_{1}^{ \pm} \\
& -\frac{z^{2} \tilde{g}}{\sqrt{2} g_{1}}\left(1+\frac{\tilde{g}^{2} z^{2}}{4 g_{1}^{2}} \frac{3 z^{4}-5 z^{2}+4}{1-z^{2}}\right) W_{2}^{ \pm} \\
\tilde{A}_{1}^{ \pm}=\frac{1}{\sqrt{2}} & \left(1-\frac{\tilde{g}^{2}}{4 g_{1}^{2}} \frac{1+z^{2}}{1-z^{2}}\right) W_{1}^{ \pm} \\
+ & \frac{1}{\sqrt{2}}\left(1+\frac{\tilde{g}^{2} z^{4}}{4 g_{1}^{2}} \frac{1+z^{2}}{1-z^{2}}\right) W_{2}^{ \pm} \\
+ & \frac{\left(1+z^{2}\right) \tilde{g}}{2 g_{1}}\left(1+\frac{\tilde{g}^{2}}{4 g_{1}^{2}} \frac{\left(1-3 z^{2}\right)\left(1+z^{4}\right)}{\left(1+z^{2}\right)}\right) W^{ \pm} \\
\tilde{A}_{2}{ }^{ \pm}= & \frac{1}{\sqrt{2}}\left(1-\frac{\tilde{g}^{2}}{4 g_{1}^{2}} \frac{1-3 z^{2}}{1-z^{2}}\right) W_{1}^{ \pm} \\
& -\frac{1}{\sqrt{2}}\left(1-\frac{\tilde{g}^{2} z^{4}}{4 g_{1}^{2}} \frac{3-z^{2}}{1-z^{2}}\right) W_{2}^{ \pm} \\
& +\frac{\left(1-z^{2}\right) \tilde{g}}{2 g_{1}}\left(1+\frac{\tilde{g}^{2}}{4 g_{1}^{2}}\left(1-3 z^{4}\right)\right) W^{ \pm}
\end{aligned}
$$


Similarly for the neutral gauge bosons the mass Lagrangian is:

$$
\mathcal{L}_{\text {mass }}^{\mathcal{N}}=\frac{1}{2} \tilde{N}^{T} \mathcal{M}_{n}^{2} \tilde{N}
$$

with $\tilde{N}^{T}=\left(\tilde{W}^{3}, \tilde{Y}, \tilde{A}_{1}^{3}, \tilde{A}_{2}^{3}\right)$ and

$$
\tilde{M}_{n}^{2}=\left(\begin{array}{cccc}
\tilde{g}^{2} f_{1}^{2} & 0 & -\tilde{g} g_{1} f_{1}^{2} & 0 \\
0 & (\tilde{g} \tan \tilde{\theta})^{2} f_{1}^{2} & 0 & -g_{1} \tilde{g} \tan \tilde{\theta} f_{1}^{2} \\
-\tilde{g} g_{1} f_{1}^{2} & 0 & g_{1}^{2}\left(f_{1}^{2}+f_{2}^{2}\right) & -g_{1}^{2} f_{2}^{2} \\
0 & -g_{1} \tilde{g} \tan \tilde{\theta} f_{2}^{2} & -g_{1}^{2} f_{2}^{2} & g_{1}^{2}\left(f_{2}^{2}+f_{1}^{2}\right)
\end{array}\right)
$$

with

$$
\tan \tilde{\theta}=\frac{\tilde{g}^{\prime}}{\tilde{g}}
$$

The corresponding non zero mass eigenvalues, up to $\mathcal{O}\left(\tilde{g}^{4} / g_{1}^{4}\right)$, are:

$$
\begin{gathered}
M_{Z}^{2}=\tilde{M}_{Z}^{2}\left(1-\frac{\tilde{g}^{2}}{g_{1}^{2}} z_{Z}\right) \\
M_{1, n}^{2}=M_{1}^{2}\left(1+\frac{\tilde{g}^{2}}{g_{1}^{2}} \frac{\sec ^{2} \tilde{\theta}}{2}\right) \\
M_{2, n}^{2}=M_{2}^{2}\left(1+\frac{\tilde{g}^{2}}{g_{1}^{2}} \frac{z^{4} \sec ^{2} \tilde{\theta}}{2}\right)
\end{gathered}
$$

where

$$
\tilde{M}_{Z}^{2}=\frac{\tilde{M}_{W}^{2}}{\cos ^{2} \tilde{\theta}}, \quad z_{Z}=\frac{1}{2} \frac{\left(z^{4}+\cos ^{2} 2 \tilde{\theta}\right)}{\cos ^{2} \tilde{\theta}}
$$

with the corresponding transformations ( $A$ is the photon field):

$$
\begin{aligned}
\tilde{W}^{3}= & \cos \tilde{\theta}\left(1-\frac{\tilde{g}^{2}}{4 g_{1}^{2} \cos ^{2} \tilde{\theta}}\left(1+z^{4}-4 \sin ^{4} \tilde{\theta}\right)\right) Z \\
& -\frac{\tilde{g}}{\sqrt{2} g_{1}}\left(1-\frac{\tilde{g}^{2}}{4 g_{1}^{2} \cos ^{2} \tilde{\theta}}\left(1-\frac{2\left(1-2 z^{2}\right) \cos 2 \tilde{\theta}}{1-z^{2}}\right)\right) Z_{1} \\
& -\frac{\tilde{g} z^{2}}{\sqrt{2} g_{1}}\left(1+\frac{\tilde{g}^{2} z^{2}}{4 g_{1}^{2} \cos ^{2} \tilde{\theta}}\left(2-3 z^{2}+\frac{2 \cos 2 \tilde{\theta}}{1-z^{2}}\right)\right) Z_{2} \\
& +\sin \tilde{\theta}\left(1-\frac{\tilde{g}^{2} \sin ^{2} \tilde{\theta}}{g_{1}^{2}}\right) A
\end{aligned}
$$




$$
\begin{aligned}
\tilde{Y}= & -\sin \tilde{\theta}\left(1-\frac{\tilde{g}^{2}}{4 g_{1}^{2} \cos ^{2} \tilde{\theta}}\left(1+z^{4}-4 \cos ^{4} \tilde{\theta}\right)\right) Z \\
& -\frac{\tilde{g} \tan \tilde{\theta}}{\sqrt{2} g_{1}}\left(1-\frac{\tilde{g}^{2}}{4 g_{1}^{2} \cos ^{2} \tilde{\theta}}\left(1+\frac{2\left(1-2 z^{2}\right) \cos 2 \tilde{\theta}}{1-z^{2}}\right)\right) Z_{1} \\
& +\frac{\tilde{g} z^{2} \tan \tilde{\theta}}{\sqrt{2} g_{1}}\left(1+\frac{\tilde{g}^{2} z^{2}}{4 g_{1}^{2} \cos ^{2} \tilde{\theta}}\left(2-3 z^{2}-\frac{2 \cos 2 \tilde{\theta}}{1-z^{2}}\right)\right) Z_{2} \\
& +\cos \tilde{\theta}\left(1-\frac{\tilde{g}^{2} \sin ^{2} \tilde{\theta}}{g_{1}^{2}}\right) A
\end{aligned}
$$

$$
\begin{aligned}
\tilde{A}_{1}^{3}= & \frac{\tilde{g}\left(z^{2}+\cos 2 \tilde{\theta}\right)}{2 g_{1} \cos \tilde{\theta}}\left(1-\frac{\tilde{g}^{2}}{4 g_{1}^{2} \cos ^{2} \tilde{\theta}}\right. \\
& \left.\left(\frac{\left.3 z^{6}-\cos ^{3} 2 \tilde{\theta}-\left(1+2 \sin ^{2} \tilde{\theta}\right) z^{4}-\left(1-4 \cos ^{4} \tilde{\theta}\right) z^{2}\right)}{\left(z^{2}+\cos 2 \tilde{\theta}\right)}\right)\right) Z
\end{aligned}
$$$$
+\frac{1}{\sqrt{2}}\left(1-\frac{\tilde{g}^{2}}{4 g_{1}^{2} \cos ^{2} \tilde{\theta}}\left(1+\frac{2 z^{2} \cos 2 \tilde{\theta}}{1-z^{2}}\right)\right) Z_{1}
$$$$
+\frac{1}{\sqrt{2}}\left(1-\frac{\tilde{g}^{2} z^{4}}{4 g_{1}^{2} \cos ^{2} \tilde{\theta}}\left(1-\frac{2 \cos 2 \tilde{\theta}}{1-z^{2}}\right)\right) Z_{2}
$$$$
+\frac{\tilde{g} \sin \tilde{\theta}}{g_{1}}\left(1-\frac{\tilde{g}^{2} \sin ^{2} \tilde{\theta}}{g_{1}^{2}}\right) A
$$

$$
\begin{aligned}
\tilde{A}_{2}^{3}= & -\frac{\tilde{g}\left(z^{2}-\cos 2 \tilde{\theta}\right)}{2 g_{1} \cos \tilde{\theta}}\left(1-\frac{\tilde{g}^{2}}{4 g_{1}^{2} \cos ^{2} \tilde{\theta}}\right. \\
& \left.\left(\frac{3 z^{6}-\left(1+2 \cos ^{2} \tilde{\theta}\right) z^{4}+\cos ^{3} 2 \tilde{\theta}-\left(1-4 \sin ^{4} \tilde{\theta}\right) z^{2}}{\left(z^{2}-\cos 2 \tilde{\theta}\right)}\right)\right) Z \\
& +\frac{1}{\sqrt{2}}\left(1-\frac{\tilde{g}^{2}}{4 g_{1}^{2} \cos ^{2} \tilde{\theta}}\left(1-\frac{2 z^{2} \cos 2 \tilde{\theta}}{1-z^{2}}\right)\right) Z_{1} \\
& -\frac{1}{\sqrt{2}}\left(1-\frac{\tilde{g}^{2} z^{4}}{4 g_{1}^{2} \cos ^{2} \tilde{\theta}}\left(1+\frac{2 \cos 2 \tilde{\theta}}{1-z^{2}}\right)\right) Z_{2} \\
& +\frac{\tilde{g} \sin \tilde{\theta}}{g_{1}}\left(1-\frac{\tilde{g}^{2} \sin ^{2} \tilde{\theta}}{g_{1}^{2}}\right) A
\end{aligned}
$$

\section{APPENDIX B: NUMERICAL VALUES}

As mentioned in Sect. IVC, we show results for three values of the $z$-parameter, $z=(0.4,1 / \sqrt{3}, 0.8)$, and various $b_{1,2^{-}}$sets. For the charged and neutral sectors, the cor- 
responding mass eigenvalues, widths and fermion-boson couplings are summarized in the following Tables:

\begin{tabular}{|c|c|c|c|c|}
\hline & $M_{(1,2), c}(\mathrm{GeV})$ & $\Gamma_{1,2}(\mathrm{GeV})$ & $a_{1}^{c}$ & $a_{2}^{c}$ \\
\hline \hline 1 & 508,1251 & $6.2,35.5$ & 0.03 & 0.20 \\
\hline 2 & 508,1251 & $6.2,28.9$ & -0.01 & -0.03 \\
\hline 3 & 1745,3001 & 183,746 & 0.15 & 0.47 \\
\hline 4 & 1745,3001 & 183,734 & -0.15 & -0.44 \\
\hline 5 & 1009,1255 & $35.3,30.5$ & 0.14 & 0.24 \\
\hline 6 & 1009,1255 & $33.1,22.2$ & -0.06 & -0.09 \\
\hline
\end{tabular}

TABLE IV: Masses, total widths and couplings to SM fermions of the charged extra gauge bosons for the six scenarios of Table I.

\begin{tabular}{|c|c|c|c|c|c|c|c|c|c|c|c|c|c|c|}
\hline & $M_{(1,2), n}(\mathrm{GeV})$ & $\Gamma_{1,2}(\mathrm{GeV})$ & $\hat{a}_{1 L}^{e}$ & $\hat{a}_{1 R}^{e}$ & $\hat{a}_{1 L}^{d}$ & $\hat{a}_{1 R}^{d}$ & $\hat{a}_{1 L}^{u}$ & $\hat{a}_{1 R}^{u}$ & $\hat{a}_{2 L}^{e}$ & $\hat{a}_{2 R}^{e}$ & $\hat{a}_{2 L}^{d}$ & $\hat{a}_{2 R}^{d}$ & $\hat{a}_{2 L}^{u}$ & $\hat{a}_{2 R}^{u}$ \\
\hline \hline 1 & 510,1251 & $6.4,36.0$ & 0.12 & 0.11 & 0.05 & 0.04 & -0.09 & -0.07 & 0.43 & -0.02 & 0.46 & -0.01 & -0.44 & 0.01 \\
\hline 2 & 510,1251 & $6.3,28.8$ & 0.02 & 0.11 & -0.05 & 0.04 & 0.01 & -0.07 & -0.08 & -0.02 & -0.07 & -0.01 & 0.07 & 0.01 \\
\hline 3 & 1736,3001 & 184,756 & 0.36 & 0.04 & 0.34 & 0.01 & -0.35 & -0.02 & 1.06 & -0.01 & 1.07 & 0.0 & -1.07 & 0.01 \\
\hline 4 & 1736,3001 & 184,742 & -0.32 & 0.04 & -0.34 & 0.01 & 0.33 & -0.02 & -1.0 & -0.01 & -0.99 & 0.0 & 1.0 & 0.01 \\
\hline 5 & 1012,1256 & $36.2,32.0$ & 0.37 & 0.08 & 0.31 & 0.03 & -0.34 & -0.06 & 0.50 & -0.05 & 0.54 & -0.02 & -0.52 & 0.04 \\
\hline 6 & 1012,1256 & $33.7,22.9$ & -0.11 & 0.08 & -0.16 & 0.03 & 0.14 & -0.06 & -0.24 & -0.05 & -0.20 & -0.02 & 0.22 & 0.04 \\
\hline
\end{tabular}

TABLE V: Masses, total widths and couplings to SM fermions of the neutral extra gauge bosons for the six scenarios of Table I. The electric charge (-e) is factorized.

Acknowledgements: E. Accomando would like to thank the Department of Physics of the University of Florence for partial financial support. We thank A. De Roeck for valuable discussions and suggestions, E. Migliore for useful informations and R. Contino for comments on LEP2 limits. D. Dominici was partially supported by MIUR under the 
contract PRIN-2006020509.

[1] C. Csaki, C. Grojean, H. Murayama, L. Pilo, and J. Terning, Phys. Rev. D69, 055006 (2004), hep-ph/0305237.

[2] K. Agashe, A. Delgado, M. J. May, and R. Sundrum, JHEP 08, 050 (2003), hep-ph/0308036.

[3] C. Csaki, C. Grojean, L. Pilo, and J. Terning, Phys. Rev. Lett. 92, 101802 (2004), hep$\mathrm{ph} / 0308038$.

[4] R. Barbieri, A. Pomarol, and R. Rattazzi, Phys. Lett. B591, 141 (2004), hep-ph/0310285.

[5] Y. Nomura, JHEP 11, 050 (2003), hep-ph/0309189.

[6] G. Cacciapaglia, C. Csaki, C. Grojean, and J. Terning, ECONF C040802, FRT004 (2004).

[7] G. Cacciapaglia, C. Csaki, C. Grojean, and J. Terning, Phys. Rev. D71, 035015 (2005), hep-ph/0409126.

[8] G. Cacciapaglia, C. Csaki, C. Grojean, and J. Terning, Phys. Rev. D70, 075014 (2004), hep-ph/0401160.

[9] R. Contino, T. Kramer, M. Son, and R. Sundrum, JHEP 05, 074 (2007), hep-ph/0612180.

[10] R. Sekhar Chivukula, D. A. Dicus, and H.-J. He, Phys. Lett. B525, 175 (2002), hep$\mathrm{ph} / 0111016$.

[11] N. Arkani-Hamed, A. G. Cohen, and H. Georgi, Phys. Rev. Lett. 86, 4757 (2001), hepth/0104005.

[12] N. Arkani-Hamed, A. G. Cohen, and H. Georgi, Phys. Lett. B513, 232 (2001), hep$\mathrm{ph} / 0105239$.

[13] C. T. Hill, S. Pokorski, and J. Wang, Phys. Rev. D64, 105005 (2001), hep-th/0104035.

[14] H.-C. Cheng, C. T. Hill, S. Pokorski, and J. Wang, Phys. Rev. D64, 065007 (2001), hepth/0104179.

[15] H. Abe, T. Kobayashi, N. Maru, and K. Yoshioka, Phys. Rev. D67, 045019 (2003), hep$\mathrm{ph} / 0205344$.

[16] A. Falkowski and H. D. Kim, JHEP 08, 052 (2002), hep-ph/0208058.

[17] L. Randall, Y. Shadmi, and N. Weiner, JHEP 01, 055 (2003), hep-th/0208120.

[18] D. T. Son and M. A. Stephanov, Phys. Rev. D69, 065020 (2004), hep-ph/0304182.

[19] J. de Blas, A. Falkowski, M. Perez-Victoria, and S. Pokorski, JHEP 08, 061 (2006), hep- 
th/0605150.

[20] R. Foadi, S. Gopalakrishna, and C. Schmidt, JHEP 03, 042 (2004), hep-ph/0312324.

[21] J. Hirn and J. Stern, Eur. Phys. J. C34, 447 (2004), hep-ph/0401032.

[22] R. Casalbuoni, S. De Curtis, and D. Dominici, Phys. Rev. D70, 055010 (2004), hep$\mathrm{ph} / 0405188$.

[23] R. S. Chivukula, E. H. Simmons, H.-J. He, M. Kurachi, and M. Tanabashi, Phys. Rev. D70, 075008 (2004), hep-ph/0406077.

[24] H. Georgi, Phys. Rev. D71, 015016 (2005), hep-ph/0408067.

[25] M. E. Peskin and T. Takeuchi, Phys. Rev. Lett. 65, 964 (1990).

[26] M. E. Peskin and T. Takeuchi, Phys. Rev. D46, 381 (1992).

[27] G. Altarelli and R. Barbieri, Phys. Lett. B253, 161 (1991).

[28] G. Altarelli, R. Barbieri, and F. Caravaglios, Int. J. Mod. Phys. A13, 1031 (1998), hep$\mathrm{ph} / 9712368$.

[29] R. Foadi, S. Gopalakrishna, and C. Schmidt, Phys. Lett. B606, 157 (2005), hep-ph/0409266.

[30] R. Casalbuoni, S. De Curtis, D. Dolce, and D. Dominici, Phys. Rev. D71, 075015 (2005), hep-ph/0502209.

[31] R. Casalbuoni, S. De Curtis, D. Dominici, and R. Gatto, Phys. Lett. B155, 95 (1985).

[32] R. Casalbuoni, S. De Curtis, D. Dominici, and R. Gatto, Nucl. Phys. B282, 235 (1987).

[33] A. Birkedal, K. Matchev, and M. Perelstein, Phys. Rev. Lett. 94, 191803 (2005), hep$\mathrm{ph} / 0412278$.

[34] A. Belyaev (2007), 0711.1919.

[35] H.-J. He et al. (2007), 0708.2588.

[36] R. Casalbuoni, S. De Curtis, D. Dominici, F. Feruglio, and R. Gatto, Int. J. Mod. Phys. A4, 1065 (1989).

[37] W. M. Yao et al. (Particle Data Group), J. Phys. G33, 1 (2006).

[38] J. Bechi, R. Casalbuoni, S. De Curtis, and D. Dominici, Phys. Rev. D74, 095002 (2006), hep-ph/0607314.

[39] R. Casalbuoni et al., Phys. Lett. B249, 130 (1990).

[40] R. Sekhar Chivukula et al., Phys. Rev. D74, 075011 (2006), hep-ph/0607124.

[41] R. Barbieri, A. Pomarol, R. Rattazzi, and A. Strumia, Nucl. Phys. B703, 127 (2004), hep$\mathrm{ph} / 0405040$. 
[42] G. Altarelli, R. Barbieri, and F. Caravaglios, Nucl. Phys. B405, 3 (1993).

[43] R. S. Chivukula, E. H. Simmons, H.-J. He, M. Kurachi, and M. Tanabashi, Phys. Rev. D72, 075012 (2005), hep-ph/0508147.

[44] R. Sekhar Chivukula, E. H. Simmons, H.-J. He, M. Kurachi, and M. Tanabashi, Phys. Rev. D72, 095013 (2005), hep-ph/0509110.

[45] R. Casalbuoni, S. De Curtis, D. Dominici, and D. Dolce, JHEP 08, 053 (2007), arXiv:0705.2510 [hep-ph].

[46] S. Dawson and C. B. Jackson, Phys. Rev. D76, 015014 (2007), hep-ph/0703299.

[47] R. Barbieri, G. Isidori, V. S. Rychkov, and E. Trincherini (2008), 0806.1624.

[48] T. Abe, S. Matsuzaki, and M. Tanabashi (2008), 0807.2298.

[49] B. W. Lee, C. Quigg, and H. B. Thacker, Phys. Rev. D16, 1519 (1977).

[50] J. M. Cornwall, D. N. Levin, and G. Tiktopoulos, Phys. Rev. Lett. 30, 1268 (1973).

[51] C. E. Vayonakis, Nuovo Cim. Lett. 17, 383 (1976).

[52] M. S. Chanowitz and M. K. Gaillard, Nucl. Phys. B261, 379 (1985).

[53] A. Ballestrero (1999), hep-ph/9911318.

[54] A. Ballestrero and E. Maina, Phys. Lett. B350, 225 (1995), hep-ph/9403244.

[55] T. Sjostrand, S. Mrenna, and P. Skands, JHEP 05, 026 (2006), hep-ph/0603175.

[56] A. Hocker, H. Lacker, S. Laplace, and F. Le Diberder, Eur. Phys. J. C21, 225 (2001), hep$\mathrm{ph} / 0104062$.

[57] J. Pumplin et al., JHEP 07, 012 (2002), hep-ph/0201195.

[58] CDF, http://www-cdfonline.fnal.gov/ops/opshelp/stores/kumac/ (2007).

[59] P. Langacker, R. W. Robinett, and J. L. Rosner, Phys. Rev. D30, 1470 (1984).

[60] M. Dittmar, Phys. Rev. D55, 161 (1997), hep-ex/9606002. 